QUARTERLY OF APPLIED MATHEMATICS

VOLUME LXX, NUMBER 2

JUNE 2012, PAGES 357-382

S 0033-569X(2012)01251-7

Article electronically published on February 29, 2012

\title{
ASYMPTOTIC STABILITY OF THE STATIONARY SOLUTION FOR A NEW MATHEMATICAL MODEL OF CHARGE TRANSPORT IN SEMICONDUCTORS
}

\author{
BY \\ A. M. BLOKHIN (Sobolev Institute of Mathematics, Novosibirsk, 630090, Russia; \\ Novosibirsk State University, Novosibirsk, 630090, Russia) \\ AND \\ D. L. TKACHEV (Sobolev Institute of Mathematics, Novosibirsk, 630090, Russia; \\ Novosibirsk State University, Novosibirsk, 630090, Russia)
}

\begin{abstract}
We study an initial boundary value problem for a system of quasilinear equations which are effectively used for finding by the stabilization method numerical stationary solutions of the hydrodynamical model of charge transport in the silicon MESFET (metal semiconductor field effect transistor). This initial boundary value problem has the following peculiarities: the PDE system is not a Cauchy-Kovalevskaya-type system; the boundary is a nonsmooth curve and has angular points; nonlinearity of the problem is mainly connected with squares of gradients of the unknown functions. By using a special representation for the solution of a model problem we reduce the original problem to a system of integro-differential equations. This allows one to prove the localin-time existence and uniqueness of a weakened solution. Using a constructed energy integral and the Schauder fixed-point theorem, we prove the global-in-time solvability of the initial boundary value problem and justify the stabilization method under additional assumptions on the problem's data.
\end{abstract}

1. Introduction. As is known, mathematical models of charge transport in semiconductors are deduced from the Boltzman transport equation by using various truncation procedures (see [1]-[8]). A system of moment equations obtained from the Boltzman transport equation with the help of the maximum entropy principle [8], [1] 13 as a

Received October 13, 2010.

2010 Mathematics Subject Classification. Primary 35G61, 35D30; Secondary 82D37.

Key words and phrases. Non-Cauchy-Kovalevskaya-type system, weakened solution, local- and globalin-time solvability, asymptotic (Lyapunov's) stability, stabilization method.

The authors are indebted to Yu. L. Trakhinin and S. A. Boyarsky for their help in the preparation of the manuscript of this paper. This work was financially supported by RFBR project, 10-01-00320-a, the interdisciplinary project of basic research SB RAS-2009 (No.91), and the project "Development of scientific potential of the Higher School" 2009-2010 (No. 2.1.1/4591).

E-mail address: blokhin@math.nsc.ru

E-mail address: tkachev@math.nsc.ru

(C)2012 Brown University Reverts to public domain 28 years from publication 


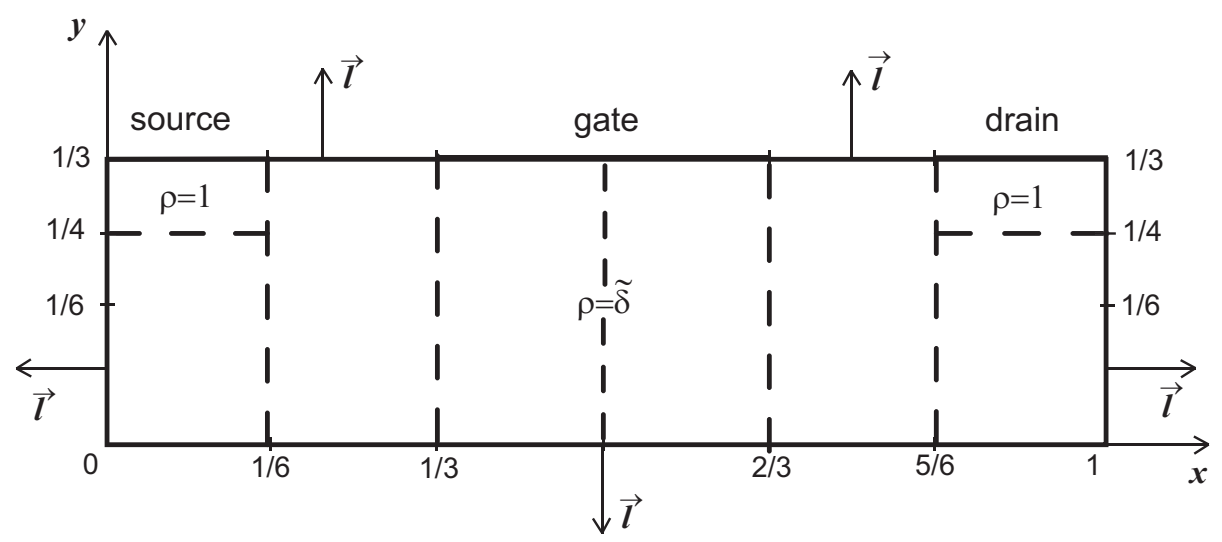

FIG. 1. Schematic sketch of the bidimensional MESFET

truncation procedure was recently suggested in [9], [10. Different mathematical and numerical aspects concerning this new hydrodynamical model were studied in [14-19] in detail.

Following [9], 10, 19], 20, we write down the quasilinear nonstationary system of the above-mentioned moment equations in the $2 \mathrm{D}$ case and in a dimensionless form (the process of reduction to a dimensionless form was detailed in [19, 20]):

$$
\left.\begin{array}{l}
R_{t}+\operatorname{div}(R \mathbf{u})=0, \\
(R \mathbf{u})_{t}+\nabla\left(\frac{2}{3} R E\right)=R\left\{\mathbf{Q}+c_{11} \mathbf{u}+c_{12} \mathbf{q}\right\}, \\
(R E)_{t}+\operatorname{div}(R \mathbf{q})=R\{(\mathbf{u}, \mathbf{Q})+c \sigma\}, \\
(R \mathbf{q})_{t}+\nabla\left(\frac{10}{9} R E^{2}\right)=R\left\{\frac{5}{3} E \mathbf{Q}+c_{21} \mathbf{u}+c_{22} \mathbf{q}\right\} .
\end{array}\right\}
$$

Here $R$ is the electron density, $E$ the electron energy, $\mathbf{u}=\left(u^{(x)}, u^{(y)}\right)$ the electron velocity (in the Cartesian coordinates $(x, y)), \mathbf{q}=\left(q^{(x)}, q^{(y)}\right)$ the energy flux, $\mathbf{Q}=\nabla \varphi=$ $\left(\varphi_{x}, \varphi_{y}\right), \sigma=\frac{2}{3} E-1 ; \varphi=\varphi(t, x, y)$ is the electric potential satisfying the Poisson equation

$$
\triangle \varphi=\varphi_{x x}+\varphi_{y y}=\beta(R-\rho)
$$

the coefficients $c, c_{11}, c_{12}, c_{21}, c_{22}$ in (1.1) are smooth functions of the energy $E$ (their explicit form is technically complicated, but for the parabolic band it can be found in [19], 20]); $\rho=\rho(x, y)$ is the doping density, and $\beta>0$ is a constant (see [19], 20]).

The mathematical model (1.1), (1.2) was used in [17, [18, 21], 22] for finding approximate stationary solutions describing the electron motion in the silicon MESFET. The detailed description of such a semiconductor transistor with electron conductivity is given in [19], 22]. Its schematic sketch is given in Figure 1 (see [22]). 
Following [17, [19], for system (1.1), (1.2) we set the following boundary conditions (see Figure 1):

$$
\begin{aligned}
& R=\left\{\begin{array}{l}
1 \text { for } y=\frac{1}{3}, 0 \leq x \leq \frac{1}{6} \text { and } \frac{5}{6} \leq x \leq 1, \\
\widetilde{N}_{g} \text { for } y=\frac{1}{3}, \frac{1}{3} \leq x \leq \frac{2}{3},
\end{array}\right. \\
& E=\frac{3}{2} \text { on } \Gamma_{0}, \\
& \varphi=\left\{\begin{array}{l}
0 \text { for } y=\frac{1}{3}, 0 \leq x \leq \frac{1}{6}, \\
\widetilde{G} \text { for } y=\frac{1}{3}, \frac{1}{3} \leq x \leq \frac{2}{3}, \\
\widetilde{B} \text { for } y=\frac{1}{3}, \frac{5}{6} \leq x \leq 1,
\end{array}\right\} \\
& (1, \nabla R)=(1, \nabla E)=(1, \nabla \varphi)=0 \text { on } \Gamma_{l}
\end{aligned}
$$

and initial data at $t=0,(x, y) \in \Omega$.

Here $\Omega=\left\{(x, y): 0<x<1,0<y<\frac{1}{3}\right\}, \Gamma_{0}=\left\{(x, y): y=\frac{1}{3}, 0 \leq x \leq \frac{1}{6}, \frac{1}{3} \leq x \leq\right.$ $\left.\frac{2}{3}, \frac{5}{6} \leq x \leq 1\right\}$ is a part of the boundary $\partial \Omega$ (see Figure 1), $\Gamma_{l}=\partial \Omega \backslash \Gamma_{0}, \mathbf{l}$ is the unit outward-pointing normal for the boundary $\partial \Omega, \widetilde{N}_{g}, \widetilde{G}, \widetilde{B}$ are constants (see [17, 22]).

Note that the constant $\widetilde{B}>0$ is called bias voltage. From Figure 1 we also see that the doping density $\rho(x, y)$ is a piecewise constant function:

$$
\rho(x, y)=\left\{\begin{array}{l}
1,(x, y) \in \bar{\Omega}_{+}, \\
\widetilde{\delta}(0<\widetilde{\delta} \leq 1),(x, y) \in \bar{\Omega} \backslash \bar{\Omega}_{+},
\end{array}\right.
$$

where the subset $\Omega_{+}$is defined as follows (see Figure 1):

$$
\Omega_{+}=\left(0, \frac{1}{6}\right) \times\left(\frac{1}{4}, \frac{1}{3}\right) \cup\left(\frac{5}{6}, 1\right) \times\left(\frac{1}{4}, \frac{1}{3}\right)
$$

REMARK 1.1. In concrete numerical calculations one usually utilizes a smooth approximation of the function $\rho(x, y)$ instead of (1.4).

REMARK 1.2. In [17, 18] a finite-difference algorithm together with the stabilization method was used for finding approximate stationary solutions of problem (1.1)-(1.3).

In 21, 22] another idea was used for constructing a numerical algorithm for finding approximate stationary solutions of the MESFET problem (1.1)-(1.3). For clarifying 
this idea we consider system (1.1), (1.2) in the stationary case:

$$
\begin{gathered}
\operatorname{div}(R \mathbf{u})=0, \\
\operatorname{div}(R \mathbf{q})=R\{(\mathbf{u}, \mathbf{Q})+c \sigma\}, \\
\nabla\left(\frac{2}{3} R E\right)=R\left\{\mathbf{Q}+c_{11} \mathbf{u}+c_{12} \mathbf{q}\right\}, \\
\nabla\left(\frac{4}{9} R E^{2}\right)=\frac{2}{5} R\left\{\frac{5}{3} E \mathbf{Q}+c_{21} \mathbf{u}+c_{22} \mathbf{q}\right\}, \\
\Delta \varphi=\operatorname{div} \mathbf{Q}=\beta(R-\rho) .
\end{gathered}
$$

We rewrite equations (1.5a) and (1.5b) as

$$
\begin{gathered}
\operatorname{div} \mathbf{u}=-(\mathbf{u}, \nabla \chi), \chi=\ln R, \\
\operatorname{div} \mathbf{q}=-(\mathbf{q}, \nabla \chi)+(\mathbf{u}, \mathbf{Q})+c \sigma .
\end{gathered}
$$

After simple calculations it follows from (1.5c), (1.5d) that

$$
\begin{gathered}
\nabla \sigma=a \mathbf{u}+b \mathbf{q} \\
\nabla \chi=m \mathbf{u}+n \mathbf{q}+\frac{1}{1+\sigma} \mathbf{Q} .
\end{gathered}
$$

Here

$$
\begin{aligned}
a & =a(E)=\frac{2}{5} \frac{c_{21}}{1+\sigma}-c_{11}, \\
b & =b(E)=\frac{2}{5} \frac{c_{22}}{1+\sigma}-c_{12}, \\
m & =m(E)=\frac{c_{11}-a}{1+\sigma} \\
n & =n(E)=\frac{c_{12}-b}{1+\sigma} .
\end{aligned}
$$

Considering (1.6), (1.7) as the system for finding components of the vectors $\mathbf{u}$, $\mathbf{q}$, we easily find that

$$
\begin{array}{r}
\mathbf{u}=F(E)\left\{\mathbf{Q}-(1+\sigma) \nabla \chi-F_{0}(E) \nabla \sigma\right\}, \\
\mathbf{q}=G(E)\left\{-\mathbf{Q}+(1+\sigma) \nabla \chi+G_{0}(E) \nabla \sigma\right\}
\end{array}
$$

where

$$
\begin{gathered}
F(E)=-\frac{c_{22}-\frac{5}{3} E c_{12}}{\operatorname{det}}, G(E)=-\frac{c_{21}-\frac{5}{3} E c_{11}}{\operatorname{det}}, \\
F_{0}(E)=1-\frac{\frac{5}{3} E c_{12}}{c_{22}-\frac{5}{3} E c_{12}}, G_{0}(E)=1-\frac{\frac{5}{3} E c_{11}}{c_{21}-\frac{5}{3} E c_{11}}, \\
\operatorname{det}=c_{11} c_{22}-c_{21} c_{12} .
\end{gathered}
$$


Applying div to (1.6), (1.7) and using equations $\left(1.5 \mathrm{a}^{\prime}\right),\left(1.5 \mathrm{~b}^{\prime}\right),(1.8),(1.9)$, we finally obtain

$$
\begin{aligned}
\Delta \sigma= & \mathcal{F}^{\sigma}(\nabla \sigma, \nabla \chi, \mathbf{Q}, \sigma)=a_{1}|\nabla \sigma|^{2}+a_{2}(\nabla \sigma, \nabla \chi) \\
& +a_{3}(\nabla \sigma, \mathbf{Q})+a_{4}(\nabla \chi, \mathbf{Q})+a_{5}|\mathbf{Q}|^{2}+b c \sigma \\
\Delta \chi= & \mathcal{F}^{\chi}(\nabla \sigma, \nabla \chi, \mathbf{Q}, \sigma, \chi, \rho)=-|\nabla \chi|^{2}+b_{1}|\nabla \sigma|^{2} \\
& +b_{2}(\nabla \sigma, \nabla \chi)+b_{3}(\nabla \sigma, \mathbf{Q})+b_{4}(\nabla \chi, \mathbf{Q})+b_{5}|\mathbf{Q}|^{2} \\
& +\frac{\beta}{1+\sigma}\left(e^{\chi}-\rho\right)+n c \sigma .
\end{aligned}
$$

Here $|\nabla \sigma|^{2}=\sigma_{x}^{2}+\sigma_{y}^{2}$, etc.;

$$
\begin{aligned}
& a_{1}=-a^{\prime} F(E) F_{0}(E)+b^{\prime} G(E) G_{0}(E), \\
& a_{2}=-1+(1+\sigma)\left\{b^{\prime} G(E)-a^{\prime} F(E)\right\}, \\
& a_{3}=a^{\prime} F(E)-b^{\prime} G(E)-b F(E) F_{0}(E), \\
& a_{4}=-b(1+\sigma) F(E), a_{5}=b F(E), \\
& b_{1}=-m^{\prime} F(E) F_{0}(E)+n^{\prime} G(E) G_{0}(E), \\
& b_{2}=(1+\sigma)\left\{n^{\prime} G(E)-m^{\prime} F(E)\right\}, \\
& b_{3}=-\frac{1}{(1+\sigma)^{2}}+m^{\prime} F(E)-n^{\prime} G(E)-n F(E) F_{0}(E), \\
& b_{4}=\frac{1}{1+\sigma}+b F(E) F_{0}(E), b_{5}=n F(E), \\
& a^{\prime}=\frac{d a}{d \sigma}=\frac{3}{2} \frac{d a}{d E}, e t c .
\end{aligned}
$$

We rewrite equation (1.5e) as

$$
\Delta \varphi=\mathcal{F}^{\varphi}(\chi, \rho)=\beta\left(e^{\chi}-\rho\right) .
$$

Thus, in the stationary case the original problem (1.1)-(1.3) can be reduced to the boundary value problem for system (1.10)-(1.12). The boundary conditions for this system follow from (1.3):

$$
\begin{aligned}
& \chi=\left\{\begin{array}{l}
0 \text { for } y=\frac{1}{3}, 0 \leq x \leq \frac{1}{6} \text { and } \frac{5}{6} \leq x \leq 1, \\
\ln \widetilde{N}_{g} \text { for } y=\frac{1}{3}, \frac{1}{3} \leq x \leq \frac{2}{3}
\end{array}\right. \\
& \sigma=0 \text { on } \Gamma_{0} ; \\
& \varphi=\left\{\begin{array}{l}
0 \text { for } y=\frac{1}{3}, 0 \leq x \leq \frac{1}{6} \\
\widetilde{G} \text { for } y=\frac{1}{3}, \frac{1}{3} \leq x \leq \frac{2}{3}, \\
\widetilde{B} \text { for } y=\frac{1}{3}, \frac{5}{6} \leq x \leq 1 ;
\end{array}\right. \\
& (1, \nabla \chi)=(1, \nabla \sigma)=(1, \nabla \varphi)=0 \text { on } \Gamma_{l} .
\end{aligned}
$$


After defining the functions $\sigma, \chi, \varphi$, the vectors $\mathbf{u}, \mathbf{q}$ are found from (1.8), (1.9).

In 21], 22] for finding approximate solutions of problem (1.10)-(1.13) the following socalled regularized mathematical model based on the stabilization method was proposed:

$$
\begin{gathered}
(\varphi-\Delta \varphi)_{t}=\Delta \varphi-\mathcal{F}^{\varphi}(\chi, \rho), \\
\varphi=\varphi(t, x, y), t>0,(x, y) \in \Omega, \\
(\sigma-\Delta \sigma)_{t}=\Delta \sigma-\mathcal{F}^{\sigma}(\nabla \sigma, \nabla \chi, \mathbf{Q}, \sigma), \\
\sigma=\sigma(t, x, y), t>0,(x, y) \in \Omega, \\
(\chi-\Delta \chi)_{t}=\Delta \chi-\mathcal{F} \chi(\nabla \sigma, \nabla \chi, \mathbf{Q}, \sigma, \chi, \rho), \\
\chi=\chi(t, x, y), t>0,(x, y) \in \Omega .
\end{gathered}
$$

We should supplement system (1.14)-(1.16) with the boundary conditions (1.13) satisfied for all $t>0$ and the initial data at $t=0$ :

$$
\begin{aligned}
& \left.\varphi\right|_{t=0}=\varphi_{0}(x, y) \\
& \left.\sigma\right|_{t=0}=\sigma_{0}(x, y) \\
& \left.\chi\right|_{t=0}=\chi_{0}(x, y) \\
& (x, y) \in \Omega
\end{aligned}
$$

REMARK 1.3. The study of stationary solutions of the boundary value problem (1.10)(1.13) is of principal importance for justifying the adequacy of the formulated hydrodynamical model of charge transport in semiconductors. In particular, as follows from standard physical arguments, after the deenergization of the transistor that corresponds to the boundary conditions (1.3) with $\widetilde{N}_{g}=1, \widetilde{B}=\widetilde{G}=0$, in a certain time the system should come to equilibrium (the so-called global thermodynamic equilibrium). Hence, the solution of problem (1.1)-(1.3) with prescribed boundary conditions and arbitrary initial data should tend to the desired stationary solution as time tends to infinity.

The results of numerical simulations [21, 22] demonstrate a fast convergence of the solution of problem (1.13)-(1.17) to the stationary solution. However, this fact requires a strict mathematical justification. The present paper is the first step in this direction.

In the present paper we study the local-in-time well-posedness of the initial boundary value problem (1.14)-(1.16), (1.13), (1.17) (Theorem 1). Also within some additional conditions we prove the existence of the weakened solution with $t \geq 0$ (Theorem 2). In this case all solutions tend to the stationary solution of the problem as $t \rightarrow \infty$ (Theorem 3). So, in this case we get the strict substantiation of the stabilization method.

2. Preliminaries. Functional spaces. Main results. Let us define the functions $Z(x)$ and $M(x)$ belonging to $C^{\infty}[0,1]$ as follows $\left(C^{\infty}[0,1]\right.$ is a class of infinitely 
differentiable functions on the segment $[0,1])$ :

$$
Z(x)=\left\{\begin{array}{l}
0 \text { if } 0 \leq x \leq \frac{1}{6}, \\
\text { a monotone decreasing function from } 0 \text { to } \widetilde{G} \text { for } \\
\frac{1}{6} \leq x \leq \frac{1}{3}, \\
\widetilde{G} \text { if } \frac{1}{3} \leq x \leq \frac{2}{3}, \\
\text { a monotone increasing function from } \widetilde{G} \text { to } \widetilde{B} \text { for } \\
\frac{2}{3} \leq x \leq \frac{5}{6}, \\
\widetilde{B} \text { if } \frac{5}{6} \leq x \leq 1 ; \\
\text { a monotone decreasing function from } 0 \text { to } \ln \widetilde{N}_{g} \text { for } \\
\frac{1}{6} \leq x \leq \frac{1}{3}, \\
\ln \widetilde{N}_{g} \text { for } \frac{1}{3} \leq x \leq \frac{2}{3}, \\
\text { a monotone increasing function from } \ln \widetilde{N}_{g} \text { to } 0 \text { for } \\
\frac{2}{3} \leq x \leq \frac{5}{6}, \\
0 \text { if } \frac{5}{6} \leq x \leq 1 .
\end{array}\right.
$$

Figure 2 illustrates the behavior of the functions $Z(x)$ and $M(x)$. Making the change of unknowns

$$
\left\{\begin{array}{l}
\varphi(t, x, y)=\varphi_{1}(t, x, y)+Z(x), \\
\sigma(t, x, y)=\sigma_{1}(t, x, y) \\
\chi(t, x, y)=\chi_{1}(t, x, y)+M(x),
\end{array}\right.
$$




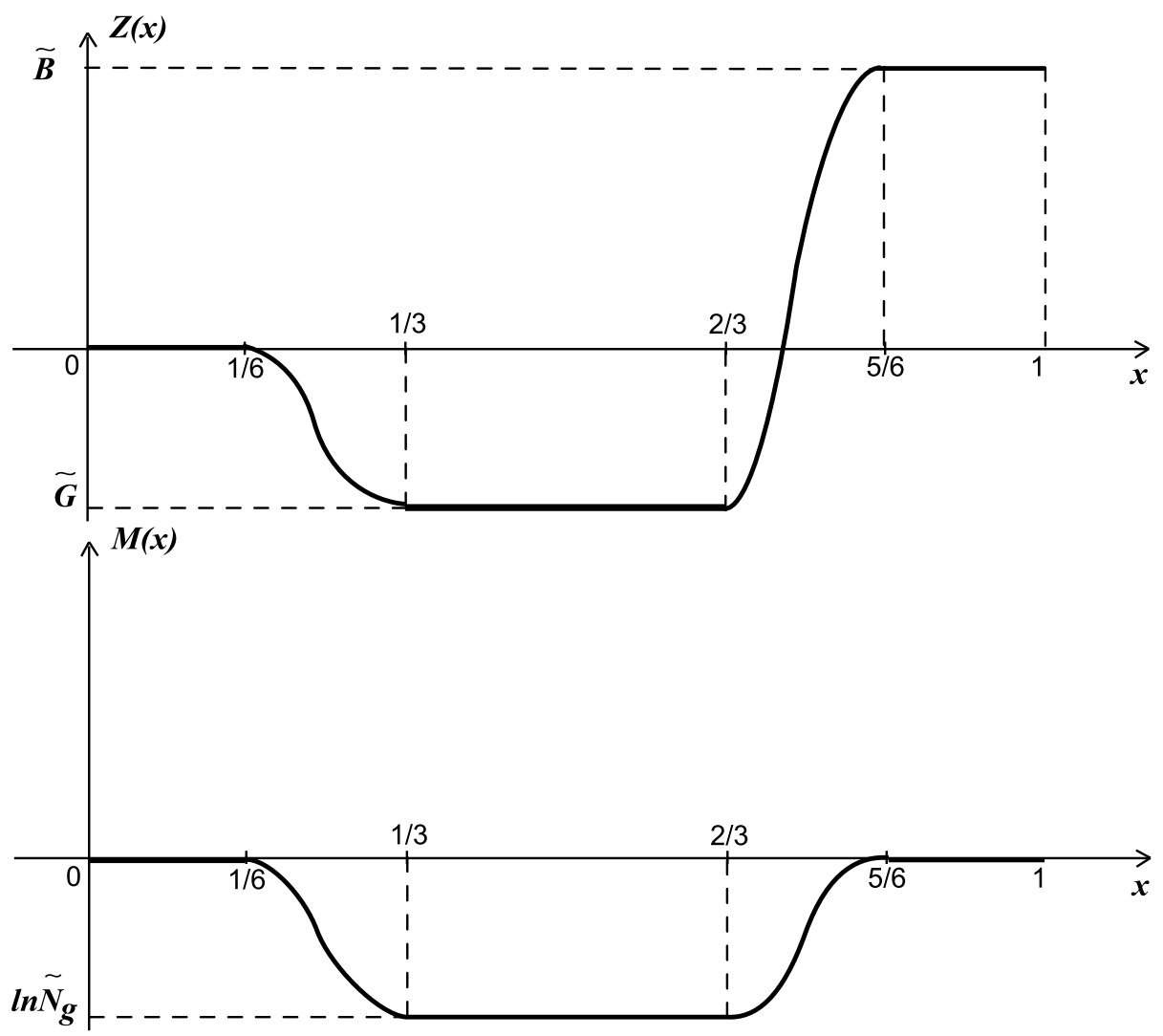

FIG. 2. Graphs of the auxiliary functions $Z(x)$ and $M(x)$

we reduce the initial boundary value problem (1.14)-(1.16), (1.13), (1.17) to

$$
\begin{gathered}
\frac{\partial}{\partial t}\left(\varphi_{1}-\Delta \varphi_{1}\right)+\varphi_{1}-\Delta \varphi_{1}=\varphi_{1}-\beta\left(e^{\chi_{1}+M(x)}-\rho\right)+Z^{\prime \prime}(x)=-\mathcal{F}^{\left(\varphi_{1}\right)}\left(\varphi_{1}, \chi_{1}\right) \\
\frac{\partial}{\partial t}\left(\sigma_{1}-\Delta \sigma_{1}\right)+\sigma_{1}-\Delta \sigma_{1}=\sigma_{1}-a_{1}\left|\nabla \sigma_{1}\right|^{2}-a_{2}\left(\nabla \sigma_{1}, \nabla \chi_{1}+\nabla M\right) \\
-a_{3}\left(\nabla \sigma_{1}, \nabla \varphi_{1}+\nabla Z\right)-a_{4}\left(\nabla \chi_{1}+\nabla M, \nabla \varphi_{1}+\nabla Z\right)-a_{5}\left|\nabla \varphi_{1}+\nabla Z\right|^{2} \\
-b c \sigma_{1}=-\mathcal{F}^{\left(\sigma_{1}\right)}\left(\sigma_{1}, \chi_{1}, \varphi_{1}\right) \\
\frac{\partial}{\partial t}\left(\chi_{1}-\Delta \chi_{1}\right)+\chi_{1}-\Delta \chi_{1}=\chi_{1}+\left|\nabla \chi_{1}+\nabla M\right|^{2}-b_{1}\left|\nabla \sigma_{1}\right|^{2} \\
-b_{2}\left(\nabla \sigma_{1}, \nabla \chi_{1}+\nabla M\right)-b_{3}\left(\nabla \sigma_{1}, \nabla \varphi_{1}+\nabla Z\right)-b_{4}\left(\nabla \chi_{1}+\nabla M, \nabla \varphi_{1}+\nabla Z\right) \\
-b_{5}\left|\nabla \varphi_{1}+\nabla Z\right|^{2}-\frac{\beta}{1+\sigma}\left(e^{\chi_{1}+M(x)}-\rho\right) \\
-n c \sigma_{1}+M^{\prime \prime}(x)=-\mathcal{F}^{\left(\chi_{1}\right)}\left(\chi_{1}, \sigma_{1}, \varphi_{1}\right) \\
(x, y) \in \Omega, t>0
\end{gathered}
$$


where the functions $\varphi_{1}(t, x, y), \sigma_{1}(t, x, y), \chi_{1}(t, x, y)$ satisfy the homogeneous boundary conditions (on $\partial \Omega$ )

$$
\begin{gathered}
\varphi_{1}=\sigma_{1}=\chi_{1}=0 \text { on } \Gamma_{0}, \\
\left(\mathbf{l}, \nabla \varphi_{1}\right)=\left(\mathbf{l}, \nabla \sigma_{1}\right)=\left(\mathbf{l}, \nabla \chi_{1}\right)=0 \text { on } \Gamma_{l}=\partial \Omega \backslash \Gamma_{0},
\end{gathered}
$$

and the initial data (1.17) become

$$
\left\{\begin{array}{l}
\left.\varphi_{1}\right|_{t=0}=\varphi_{0}(x, y)-Z(x)=\widetilde{\varphi}_{0}(x, y) \\
\left.\sigma_{1}\right|_{t=0}=\sigma_{0}(x, y) \\
\left.\chi_{1}\right|_{t=0}=\chi_{0}(x, y)-M(x)=\tilde{\chi}_{0}(x, y) .
\end{array}\right.
$$

Note that the coefficients $b, c, n, a_{i}, b_{i}(i=\overline{1,5})$ stay unchanged because they depend only on $\sigma_{1}(t, x, y)$. Below we will drop the index 1 by the unknown functions $\varphi_{1}, \sigma_{1}, \chi_{1}$ as well as tildes in the initial data (2.5).

Introducing the notation

$$
\begin{gathered}
\Theta_{\varphi}=\varphi-\Delta \varphi, \\
\Theta_{\sigma}=\sigma-\Delta \sigma, \\
\Theta_{\chi}=\chi-\Delta \chi,
\end{gathered}
$$

we can compactly write problem (2.2)-(2.5) as follows:

$$
\begin{gathered}
\frac{\partial}{\partial t} \Theta_{\varphi}+\Theta_{\varphi}=-\mathcal{F}^{(\varphi)}(\varphi, \chi), \\
\frac{\partial}{\partial t} \Theta_{\sigma}+\Theta_{\sigma}=-\mathcal{F}^{(\sigma)}(\sigma, \chi, \varphi), \\
\frac{\partial}{\partial t} \Theta_{\chi}+\Theta_{\chi}=-\mathcal{F}^{(\chi)}(\chi, \sigma, \varphi), \\
(x, y) \in \Omega, t>0 ; \\
\varphi=\sigma=\chi=0 \text { on } \Gamma_{0} ; \\
(\mathbf{l}, \nabla \varphi)=(\mathbf{l}, \nabla \sigma)=(\mathbf{l}, \nabla \chi)=0 \text { on } \Gamma_{l} ; \\
\left\{\begin{array}{l}
\left.\varphi\right|_{t=0}=\varphi_{0}(x, y), \\
\left.\sigma\right|_{t=0}=\sigma_{0}(x, y), \\
\left.\chi\right|_{t=0}=\chi_{0}(x, y) .
\end{array}\right.
\end{gathered}
$$

Let us recall the definitions of some well-known functional spaces:

$L_{\infty}(\Omega)$ is the Banach space of Lebesgue measurable functions $u(x, y)$ such that

$$
\|u\|_{L_{\infty}(\Omega)}=\operatorname{ess}_{(x, y) \in \Omega}|u(x, y)|<\infty ;
$$

$H^{1}(\Omega)$ is the well-known Sobolev space that is the Hilbert space of functions $u(x, y) \in$ $L_{2}(\Omega)$ having first-order distributional derivatives and endowed with the norm

$$
\|u\|_{H^{1}(\Omega)}=\sqrt{\sum_{0 \leq|\alpha| \leq 1} \int_{\Omega}\left|\frac{\partial^{\alpha}}{\partial^{\alpha_{1}} x \partial^{\alpha_{2}} y} u\right|^{2} d x d y}
$$


$\stackrel{\circ}{H}^{1}(\Omega)$ is the closed subspace of $H^{1}(\Omega)$ containing the functions whose trace is zero on the boundary $\Gamma=\partial \Omega$;

$H^{-1}\left(R^{2}\right)$ is the space of distributions on $R^{2}$ with the norm

$$
\|u\|_{H^{-1}\left(R^{2}\right)}=\left\|\left(1+|\xi|^{2}\right)^{-1 / 2} \widehat{u}\right\|_{L_{2}\left(R_{2}\right)}
$$

making it a Hilbert space $\left(\widehat{u}\left(\xi_{1}, \xi_{2}\right)\right.$ is the Fourier transform of a function $\left.u(x, y)\right)$.

As is known [23], 24], the dual space to $H^{1}(\Omega)$ coincides with $H_{\bar{\Omega}}^{-1}\left(R^{2}\right)$, which is the subspace of distributions from $H^{-1}\left(R^{2}\right)$ with supports in $\bar{\Omega}$, i.e., $\left[H^{1}(\Omega)\right]^{*}=H_{\bar{\Omega}}^{-1}\left(R^{2}\right)$. Nonsmoothness of the boundary $\Omega$ is not important in our case because any function from $H^{1}(\Omega)$ can be continued as a compactly supported function into a domain with a smooth boundary [25]. The space $H_{\bar{\Omega}}^{-1}\left(R^{2}\right)$ is identified as the closure of the space $\widetilde{D}(\Omega)$ in the topology of $H^{-1}\left(R^{2}\right)$, where $\widetilde{D}(\Omega)$ is the space of compactly supported regular functions on $\Omega$ continued by zero outside $\Omega$ (see $[23)$. The spaces $H^{-1}(\Omega)\left(=\left[\stackrel{\circ}{H}^{1}(\Omega)\right]^{*}\right)$ and $H_{\bar{\Omega}}^{-1}\left(R^{2}\right)$ are connected as follows: $H^{-1}(\Omega)$ is isomorphic to $H_{\bar{\Omega}}^{-1}\left(R^{2}\right) / H_{\Gamma}^{-1}\left(R^{2}\right)$, where $H_{\Gamma}^{-1}\left(R^{2}\right)$ is the subspace of distributions from $H_{\bar{\Omega}}^{-1}\left(R^{2}\right)$ with supports on $\Gamma=\partial \Omega$, i.e., $H_{\Gamma}^{-1}\left(R^{2}\right) \perp H_{0}^{1}(\Omega)$ or $\forall f \in H_{\Gamma}^{-1}\left(R^{2}\right)$ and $\forall \varphi \in H_{0}^{1}(\Omega)\langle f, \varphi\rangle=0$, where " $\langle\cdot, \cdot \cdot\rangle$ " denotes the duality brackets. Thus, there is an essential difference between the spaces $H^{-1}(\Omega)$ and $H_{\bar{\Omega}}^{-1}\left(R^{2}\right)$.

The space $H^{1 / 2}\left(\Gamma_{0}\right)$ is the Hilbert space of traces of functions $u \in H^{1}(\Omega)$ :

$$
\|v\|_{H^{1 / 2}\left(\Gamma_{0}\right)}=\inf \left\{\|u\|_{H^{1}(\Omega)}:\left.u\right|_{\Gamma_{0}}=v\right\},
$$

and $H^{-1 / 2}\left(\Gamma_{l}\right)$ is the dual space to $H^{1 / 2}\left(\Gamma_{l}\right)$ with respect to the scalar product in $L_{2}\left(\Gamma_{l}\right)$.

Now consider spaces of continuous functions [26], 27]. A function $u(x, y)=u(\mathcal{P})$ is called uniformly Hölder continuous of index $\alpha$ in the domain $\Omega$ if

$$
[u]_{\alpha ; \Omega}=\sup _{\mathcal{P}_{1}, \mathcal{P} \in \Omega, \mathcal{P}_{1} \neq \mathcal{P}_{2}} \frac{\left|u\left(\mathcal{P}_{1}\right)-u\left(\mathcal{P}_{2}\right)\right|}{\left|\mathcal{P}_{1}-\mathcal{P}_{2}\right|^{\alpha}} \quad(0<\alpha \leq 1)
$$

is bounded, where

$$
\mathcal{P}_{1}=\left(x_{1}, y_{1}\right), \quad \mathcal{P}_{2}=\left(x_{2}, y_{2}\right), \quad\left|\mathcal{P}_{1}-\mathcal{P}_{2}\right|^{\alpha}=\left\{\left(x_{1}-x_{2}\right)^{2}+\left(y_{1}-y_{2}\right)^{2}\right\}^{\frac{\alpha}{2}} .
$$

It is called locally Hölder continuous of index $\alpha$ in $\Omega$ if $u$ is uniformly continuous of index $\alpha$ on all compact subsets of the domain $\Omega$.

A subspace of the Banach space $C^{k}(\bar{\Omega})$ containing functions whose $k$ th derivatives ( $k \geq 0$ is integer) are uniformly Hölder continuous of index $\alpha$ in the domain $\Omega$ is called the Hölder subspace $C^{k, \alpha}(\bar{\Omega})$. The Hölder subspace is a Banach space. The norms in the spaces $C^{k}(\bar{\Omega})$ and $C^{k, \alpha}(\bar{\Omega})$ are defined as follows:

$$
\begin{gathered}
\|u\|_{C^{k}(\bar{\Omega})}=\sum_{|\beta|=0}^{k} \sup _{\Omega}\left|D^{\beta} u(x, y)\right|, \beta=\left(\beta_{1}, \beta_{2}\right), \\
D_{\beta}=\frac{\partial^{|\beta|}}{\partial x^{\beta_{1}} \partial y^{\beta_{2}}},|\beta|=\beta_{1}+\beta_{2}, \beta_{1,2} \geq 0 \quad \text { are integer, } \\
\|u\|_{C^{k, \alpha}(\bar{\Omega})}=\|u\|_{C^{k}(\bar{\Omega})}+\sum_{|\beta|=k}\left[D^{\beta} u(x, y)\right]_{\alpha ; \Omega} .
\end{gathered}
$$


The linear space $C^{k, \alpha}(\Omega)$ is the set of functions belonging to $C^{k, \alpha}\left(\bar{\Omega}^{\prime}\right)$ for any compact subset $\bar{\Omega}^{\prime} \subset \Omega$.

Let $D$ be a certain Banach space. By $A C([0, T] ; D)$ and $C^{1}([0, T] ; D)$ we denote the Banach space of absolutely continuous functions with respect to $t$ with values in $D$ and the Banach space of continuously differentiable functions with respect to $t$ with values in $D$ respectively ( $t$-derivatives are understood in a classical sense). The space $L_{\infty}([0, T] ; D)$ is defined analogously.

Let us now return to the statement of problem (2.2)-(2.5) (or (2.6)-(2.9)) and define the following closed subspaces in the space $H^{1}(\Omega)$ :

$$
\begin{gathered}
P=\left\{u \in H^{1}(\Omega)|u|_{\Gamma_{0}}=0\right\}, \\
N=\left\{u \in H^{1}(\Omega) \mid u=0 \text { on } \Gamma_{0},(\mathbf{l}, \nabla u)=0 \text { on } \Gamma_{l}\right\}, \quad \text { i.e., }\left.u\right|_{\Gamma_{0}} \in H^{1 / 2}\left(\Gamma_{0}\right), \\
\left.(\mathbf{l}, \nabla u)\right|_{\Gamma_{l}} \in H^{-1 / 2}\left(\Gamma_{l}\right) .
\end{gathered}
$$

Following [28, we now give the notions of a strong generalized solution and a weakened solution.

Definition 1. A vector function $U=(\varphi, \sigma, \chi)^{T}$ from $\left[C^{1}\left([0, T], N \cap L_{\infty}(\Omega)\right)\right]^{3}$ satisfying the conditions

$$
\begin{gathered}
\left\langle U^{\prime}-\Delta U^{\prime}+U-\Delta U+\mathcal{F}, W\right\rangle=0 \\
\forall W=\left(W_{1}, W_{2}, W_{3}\right)^{T} \in\left[N \cap L_{\infty}(\Omega)\right]^{3}, \forall t \in[0, T], \\
U(0)=\left(\varphi_{0}, \sigma_{0}, \chi_{0}\right)^{T} \in\left[N \cap L_{\infty}(\Omega)\right]^{3}
\end{gathered}
$$

is called the strong generalized solution of problem $(2.2)-(2.5)$, where

$$
\mathcal{F}=\left(\begin{array}{c}
\mathcal{F}^{(\varphi)}(\varphi, \chi) \\
\mathcal{F}^{(\sigma)}(\sigma, \chi, \varphi) \\
\mathcal{F}^{(\chi)}(\chi, \sigma, \varphi)
\end{array}\right),
$$

$\left[C^{1}\left([0, T],\left[N \cap L_{\infty}(\Omega)\right]^{3}\right)\right]^{3}$ and $\left[N \cap L_{\infty}(\Omega)\right]^{3}$ are Cartesian products of corresponding spaces, and the linear form in (2.13) ("duality brackets") reads

$$
\begin{gathered}
\left\langle U^{\prime}-\Delta U^{\prime}+U-\Delta U+\mathcal{F}, W\right\rangle=\left\langle\varphi^{\prime}-\Delta \varphi^{\prime}+\varphi-\Delta \varphi\right. \\
\left.+\mathcal{F}^{(\varphi)}(\varphi, \chi), W_{1}\right\rangle+\left\langle\sigma^{\prime}-\Delta \sigma^{\prime}+\sigma-\Delta \sigma+\mathcal{F}^{(\sigma)}(\sigma, \chi, \varphi), W_{2}\right\rangle \\
+\left\langle\chi^{\prime}-\Delta \chi^{\prime}+\chi-\Delta \chi+\mathcal{F}^{(\chi)}(\chi, \sigma, \varphi), W_{3}\right\rangle
\end{gathered}
$$

(in the right-hand side the duality brackets are already used for scalar functions).

REMARK 2.1. In our case expression (2.13) allows us to use a formula of integration by parts, if $W \in\left[P \cap L_{\infty}(\Omega)\right]^{3}$. In monograph [26] a generalized solution is found from $H^{1}(\Omega)$ for the second-order equation, when boundary conditions includes first-order derivatives. The case of the high-order equation is studied in the classical monograph [23]. Also in this monograph is studied a question, in what meaning can we understand the boundary conditions (2.3), (2.4).

REMARK 2.2. As is known [26], for planar strictly Lipshitz domains $\Omega$ (as in our case) the spaces

$$
\stackrel{*}{H}(\Omega)=H^{1}(\Omega) \cap L_{\widetilde{m}}(\Omega)
$$


and $H^{1}(\Omega)$ coincide $\left(L_{\widetilde{m}}(\Omega)\right.$ is the Lebesgue space and $\widetilde{m} \geq 1$ is an arbitrary number). Therefore, the condition $U \in\left[C^{1}\left([0, T], N \cap L_{\infty}(\Omega)\right)\right]^{3}$ is close to a natural condition $U \in\left[C^{1}([0, T], N)\right]^{3}$.

We define $\widetilde{C}^{1}(\bar{\Omega})=\left\{u \in C^{1}(\bar{\Omega}) \mid u=0\right.$ on $\Gamma_{0}, \frac{\partial u}{\partial n}=0$ on $\left.\Gamma_{l}\right\}$, which is a closed subspace in $C^{1}(\bar{\Omega})$.

Definition 2. A strong generalized solution $U=(\varphi, \sigma, \chi)^{T}$ of problem $(2.2)-(2.5)$ from $\left[C^{1}\left([0, T], \widetilde{C}^{1}(\bar{\Omega})\right)\right]^{3}$ is called the weakened solution of this problem.

We are now in a position to state the following local-in-time existence theorem in the class of weakened solutions.

Theorem 1. For any initial data $U(0) \in\left[\widetilde{C}^{1}(\bar{\Omega})\right]^{3}$ there is a maximal time $T_{0}>0$ such that for all $t<T_{0}$ there exists a unique weakened solution of problem (2.2)-(2.5). Moreover, either $T_{0}=+\infty$ or $T_{0}<+\infty$ and in the last case,

$$
\limsup _{t \uparrow T_{0}} \sup _{(x, y) \in \Omega}\left\|\nabla_{x, y} U\right\|=+\infty .
$$

This result can be strengthened under additional assumptions on the problem's data.

TheOREM 2. Let the quantities $\|\widetilde{\delta}\|_{L_{\infty}(\Omega)}=\|\rho-1\|_{L_{\infty}(\Omega)},\|U(0)\|_{\left[\widetilde{C}^{1}(\bar{\Omega})\right]^{3}}, \widetilde{G}, \widetilde{B}$ and $\ln \widetilde{N}_{g}$ be small enough and $U(0) \in\left[H^{2}(\Omega)\right]^{3}$. Then the weakened solution of problem (2.2)-(2.5) exists for all $t \geq 0$.

Theorem 3. Let all the assumptions of Theorem 2 be satisfied. Then all the weakened solutions of problem (2.2)-(2.5) converge to the stationary solution $U^{*}(x, y)$ of this problem as time tends to infinity, i.e.,

$$
\left\|U(t, x, y)-U^{*}(x, y)\right\|_{\left[\widetilde{C}^{1}(\bar{\Omega})\right]^{3}} \rightarrow 0 \text { as } t \rightarrow \infty .
$$

REMARK 2.3. The degree of smallness of the quantities $\|\widetilde{\delta}\|_{L_{\infty}(\Omega)},\|U(0)\|_{\left[\widetilde{C}^{1}(\bar{\Omega})\right]^{3}}, \widetilde{G}$, $\widetilde{B}$ and $\ln \widetilde{N}_{g}$ will be found during the proof of Theorems 2 and 3. The main requirement for these quantities is the validity of inequality (4.30) below.

3. The proof of Theorem 1. Let us first modify a little bit the statement of problem (2.2)-(2.5) keeping the previous notation and assuming that the function $\rho(x, y)$ is not piecewise constant but belongs to $C^{1}(\bar{\Omega})$.

Consider the following matrix differential operator $A$ :

$$
A=\left(\begin{array}{ccc}
-\Delta+I & 0 & 0 \\
0 & -\Delta+I & 0 \\
0 & 0 & -\Delta+I
\end{array}\right): N^{3} \rightarrow\left[H_{\bar{\Omega}}^{-1}\left(R^{2}\right)\right]^{3},
$$

where $I$ is the identity operator. Then

$$
\begin{aligned}
\left\langle A U_{1}-A U_{2}, U_{1}-U_{2}\right\rangle= & \sum_{i=1}^{3}\left\|\nabla u_{i}^{(1)}-\nabla u_{i}^{(2)}\right\|_{\left[L_{2}(\Omega)\right]^{3}}^{2}+\left\|U_{1}-U_{2}\right\|_{L_{2}(\Omega)}^{2} \\
& \geq\left\|U_{1}-U_{2}\right\|_{\left[H^{1}(\Omega)\right]^{3}}^{2}
\end{aligned}
$$


and $U_{k}=\left(u_{1}^{(k)}, u_{2}^{(k)}, u_{3}^{(k)}\right), k=1,2$. It follows from inequality (3.2) that

$$
\begin{gathered}
\left\|A^{-1} Z_{1}-A^{-1} Z_{2}\right\|_{N^{3}} \leq\left\|Z_{1}-Z_{2}\right\|_{\left[H_{\bar{\Omega}}^{-1}\left(R^{2}\right)\right]^{3}}, \\
Z_{1}, Z_{2} \in \operatorname{Im} A \subset\left[H_{\bar{\Omega}}^{-1}\left(R^{2}\right)\right]^{3} .
\end{gathered}
$$

Hence, $A$ has an inverse operator that is Lipshitz continuous and the Lipschitz constant is equal to unity.

Taking into account the definition of the strong generalized solution (see Definition 1), problem (2.6)-(2.9) can be rewritten in the compact form

$$
\begin{aligned}
A U^{\prime}+A U & =-\mathcal{F}, \\
U(0)=\left(\varphi_{0}, \sigma_{0}, \chi_{0}\right)^{T} & \in\left[N \cap L_{\infty}(\Omega)\right]^{3} .
\end{aligned}
$$

Since we seek solutions of problem (3.4) in a weakened sense, the following chains of inclusions take place:

$$
\begin{gathered}
U^{\prime} \in\left[C\left([0, T] ; \widetilde{C}^{1}(\bar{\Omega})\right)\right]^{3} \subset\left[C\left([0, T] ;\left[N \cap L_{\infty}(\Omega)\right]^{3}\right)\right]^{3} \\
\mathcal{F} \in\left[C^{1}([0, T] ; C(\bar{\Omega}))\right]^{3} \subset\left[C^{1}\left([0, T] ; H_{\bar{\Omega}}^{-1}\left(R^{2}\right)\right)\right]^{3} .
\end{gathered}
$$

Then, from (3.4) we obtain

$$
\begin{aligned}
& U^{\prime}+U=-A^{-1}(\mathcal{F}), \\
& U(0)=\left(\varphi_{0}, \sigma_{0}, \chi_{0}\right)^{T} .
\end{aligned}
$$

We seek a solution of problem $(3.5)-(3.6)$ in the class $\left[C^{1}\left([0, T] ; \widetilde{C}^{1}(\bar{\Omega})\right)\right]^{3}$.

We prove that the operator $A^{-1}$ is an "integral" operator. For this purpose we use some results obtained for the following scalar problem [29]:

$$
\begin{gathered}
\Delta \varphi=f(x, y), \quad(x, y) \in \Omega ; \\
\left.\varphi\right|_{\Gamma_{0}}=0 ; \\
(\mathbf{l}, \nabla \varphi)=0 \text { on } \Gamma_{l} .
\end{gathered}
$$

Theorem (29]). Let the function $f(x, y) \in C^{\alpha}(\Omega)$ be bounded on $\Omega$, i.e.,

$$
\sup _{\Omega}|f(x, y)| \leq M<\infty .
$$

Then the boundary value problem (3.7)-(3.9) has the unique solution $\varphi(x, y) \in C^{2, \alpha}(\Omega) \cap$ $C^{1, \alpha}(\bar{\Omega})$, which can be represented in the form

$$
\begin{aligned}
\varphi(x, y)= & \frac{1}{2 \pi} \iint_{\Omega} f(\xi, \eta) \ln |z-\xi| d \xi d \eta+\operatorname{Re} \int_{\frac{1}{m}}^{s n\left(\frac{k}{3 a}(x-3 a+i y), m\right)} r(\zeta) d \zeta \\
& -\frac{1}{4 \pi} \iint_{\Omega} f(\xi, \eta) \ln \left(\xi^{2}+(2 a-\eta)^{2}\right) d \xi d \eta, z=x+i y, \quad \zeta=\xi+i \eta,
\end{aligned}
$$

where the elliptic Jacobi function $s n(\omega, m)$ is the inverse function to an elliptic integral of the first kind [30, $r(\zeta)$ is the Cauchy integral defined in [29], and $k, m, a$ are certain parameters. Solution (3.10) satisfies the a priori estimate

$$
\|\varphi\|_{C^{1, \alpha}(\bar{\Omega})} \leq C \sup _{\Omega}|f(x, y)|, 0<\alpha<1
$$

and the constant $C>0$ is finally determined by the constant $\alpha$. 
REMARK 3.1. In the theorem above it is assumed that the domain $\Omega$ is a rectangle: $\Omega\{(x, y) \mid 0<x<6 a, 0<y<2 a\}$. In our case $a=\frac{1}{6}$.

Consider now the following model problem for problem (2.2)-(2.5). We seek for a solution of the scalar equation

$$
-\Delta u+u=f(x, y),(x, y) \in \Omega
$$

satisfying the mixed boundary conditions

$$
\begin{gathered}
\left.u\right|_{\Gamma_{0}}=0, \\
(\mathbf{l}, \nabla u)=0 \text { on } \Gamma_{l} .
\end{gathered}
$$

Let $f \in L_{2}(\Omega)$. A function $u \in N$ will be called the generalized solution of problem (3.12)-(3.14) if the following integral identity holds for any function $v \in N$ :

$$
\int_{\Omega}(\nabla u, \nabla v) d x d y+\int_{\Omega} u v d x d y=\int_{\Omega} f v d x d y .
$$

There are no other conditions to be satisfied by $u$.

Using the well-known Friedrichs inequality [26, 31]

$$
\|u\|_{L_{2}(\Omega)} \leq C|| \nabla u \|_{L_{2}(\Omega)}
$$

with a constant $C>0$ and standard arguments connected with the introduction of an equivalent norm and the Riesz representation theorem [26], 32], we conclude that finding a generalized solution of problem (3.12)-(3.14) is equivalent to solving the following second-kind Fredholm equation:

$$
u+L u=L f, u \in N,
$$

where $L$ is a completely continuous operator.

On the other hand, we assume that $f \in C(\bar{\Omega})$ (if the assumptions of the theorem are fulfilled, then the right-hand parts of system (2.2) (or (2.6)) indeed belong to this class).

Since the subspace $H^{1}(\Omega)$ is embedded into $L^{q}(\Omega)$ for $q \in(2, \infty)$ (see [28]), the function $u(x, y)$ defined in (3.10) belongs to $C^{1, \alpha}(\bar{\Omega}), q=\frac{2}{1-\alpha}, 0<\alpha<1$ (see [27]), and estimate (3.11) takes place. Thus, in this case, equation (3.12) is equivalent to the integral Fredholm equation in the space $C^{1, \alpha}(\bar{\Omega})$ (see [27] also):

$$
u-\widetilde{\Delta}^{(-1)} u=-\widetilde{\Delta}^{(-1)} f,
$$

and the operator $\widetilde{\Delta}^{(-1)}$ is defined by formula (3.10).

Let us now improve the Friedrichs inequality (3.16) in our case.

Lemma. For $u \in H^{1}(\Omega)$ with $\left.u\right|_{\Gamma_{0}}=0$ the following inequality holds:

$$
\|u\|_{L_{2}(\Omega)} \leq \frac{\sqrt{13}}{6 \sqrt{2}}\|\nabla u\|_{L_{2}(\Omega)} .
$$

Proof. Let $u \in C^{1}(\bar{\Omega})$ and $\left.u\right|_{\Gamma_{0}}=0$. Then

$$
u(x, y)=\int_{\left(\frac{1}{2}, y\right)}^{(x, y)} \frac{\partial u}{\partial x} d x+\int_{\left(\frac{1}{2}, \frac{1}{3}\right)}^{\left(\frac{1}{2}, y\right)} \frac{\partial u}{\partial y} d y .
$$


Using the Young inequality, one gets

$$
\int_{\Omega}|u(x, y)|^{2} d x d y \leq \max \left\{\frac{1}{8}(1+\varepsilon), \frac{1}{18}\left(1+\frac{1}{\varepsilon}\right)\right\} \int_{\Omega}\|\nabla u\|^{2} d x d y .
$$

For $\varepsilon=\frac{4}{9}$ the coefficient in the right-hand part of the last inequality has the minimal value $\frac{13}{72}$. Then

$$
\|u\|_{L_{2}(\Omega)} \leq \frac{\sqrt{13}}{6 \sqrt{2}}\|\nabla u\|_{L_{2}(\Omega)} .
$$

Since the subspace $C^{1}(\bar{\Omega})$ with the additional condition $\left.u\right|_{\Gamma_{0}}=0$ is dense in the subspace $\left\{u \in H^{1}(\Omega)|u|_{\Gamma_{0}}=0\right\}$, we deduce the desired estimate (3.19).

It follows from the Friedrichs inequality that the discrete spectrum of the Laplace operator $\Delta$ defined on the subspace $N$ lies on the half-line $\left(-\infty,-\frac{72}{13}\right]$.

REMARK 3.2. In the present work we do not want to find the minimal eigenvalue of the operator $\Delta$ for the domain of definition described above with a highest possible accuracy. This is a separate problem.

Then, the solution of the Fredholm equation (3.18) in the space $C^{1, \alpha}(\bar{\Omega})$ is represented by the regularly convergent Neumann series

$$
u=-\sum_{k=1}^{n}\left[\widetilde{\Delta}^{(-1)}\right]^{k} f
$$

and the a priori estimate

$$
\|u\|_{C^{1, \alpha}(\bar{\Omega})} \leq \widetilde{C} \sup _{\Omega}|f(x, y)|, \widetilde{C}>0
$$

holds.

Thus, the operator $A^{-1}$ is indeed "integral" in the sense that its diagonal elements are decomposed into convergent power series of the integral operator staying in the right-hand part of representation (3.21). Returning to (3.5), we can say that the generalized Green matrix has been found. It is the kernel of the integral representation of the operator $A^{-1}$.

Continuing the proof of the theorem, we rewrite problem (3.5), (3.6) as

$$
U(t, x, y)=e^{-t} U(0)-\int_{0}^{t} e^{-(t-\xi)} A^{-1}(\mathcal{F}) d \xi \equiv H(U) .
$$

To prove the local-in-time existence of solutions of equation $(3.23)$ in the space $\left[L_{\infty}([0, T]\right.$; $\left.\widetilde{C}^{1}(\bar{\Omega})\right]^{3}$ we use the fixed-point argument $[28$. For this purpose we consider a convex bounded subset in this space:

$$
\begin{aligned}
B_{R}=\left\{U \in\left[L_{\infty}\left([0, T] ; \widetilde{C}^{1}(\bar{\Omega})\right)\right]^{3} \mid\right. & \\
& \left.\|U\|_{T}=\operatorname{ess}_{\sup } \operatorname{su}_{t \in(0, T)} \sup _{(x, y) \in \Omega}\left(\sum_{i=1}^{3}\left\|\nabla u_{i}\right\|^{2}\right)^{1 / 2} \leq R\right\} .
\end{aligned}
$$

Introduce the notation

$$
|U|=\sup _{(x, y) \in \Omega}\left(\sum_{i=1}^{3}\left\|\nabla u_{i}\right\|^{2}\right)^{1 / 2} .
$$


We prove that the operator $H(U)$ defined in (3.23) acts from $B_{R}$ to $B_{R}$ and is a contraction mapping on the above set. Indeed, using the formula $\sigma=\frac{2}{3} E-1$, the a priori estimate (3.11), and representation (3.20), we get the estimate

$$
\|H(U)\|_{T} \leq|U(0)|+T \mu(R)|| U \|_{T}+T \cdot|K|,
$$

which guarantees that our vector function falls into the set $B_{R}$ for a suitable choice of initial data $U(0)$ and a sufficiently short time $T$. Here $K(x, y)$ is the vector of right-hand parts:

$$
K=A^{-1}\left(\begin{array}{c}
\beta\left(\rho-e^{M(x)}\right)+Z^{\prime \prime}(x) \\
0 \\
M^{\prime \prime}(x)
\end{array}\right) .
$$

In $(3.24), \mu(R)$ is a positive function.

It follows from (3.23) and the well-known Hadamard lemma 33 that

$$
\left\|H\left(U_{1}\right)-H\left(U_{2}\right)\right\|_{T} \leq T \cdot \mu_{1}(R)\left\|\widetilde{U}_{1}-\widetilde{U}_{2}\right\|_{T}, \widetilde{U}_{1}, \widetilde{U}_{2} \in B_{R} .
$$

Hence, for $T \cdot \mu(R)<1$ the operator $H(U)$ is a contraction mapping on $B_{R}$ [33. Thus, equation (3.23) has a unique solution for sufficiently short times $t$.

Using a standard algorithm of continuation of solutions along the time axis (continuation principle), we obtain the existence of such a maximal time $T_{0}>0$ that the solution from $\left[L^{\infty}\left([0, T] ; \widetilde{C}^{1}(\bar{\Omega})\right)\right]^{3}$ is unique for any $T \in\left(0, T_{0}\right)$ and either $T_{0}=+\infty$ or $T_{0}<+\infty$, and in the last case,

$$
\limsup _{t \uparrow T_{0}}|U|=+\infty .
$$

The properties of smoothing of the operator $H(U)$ allow one to conclude that the vector function

$$
\begin{gathered}
U(t, x, y) \in\left[C^{(1)}\left([0, T] ; \widetilde{C}^{1}(\bar{\Omega})\right)\right]^{3}, T<T_{0} \text { because } \\
H(U):\left[L_{\infty}\left([0, T] ; \widetilde{C}^{1}(\bar{\Omega})\right)\right]^{3} \rightarrow\left[A C\left([0, T] ; \widetilde{C}^{1}(\bar{\Omega})\right)\right]^{3}, \\
H(U):\left[A C\left([0, T] ; \widetilde{C}^{1}(\bar{\Omega})\right)\right]^{3} \rightarrow\left[C^{1}\left([0, T], \widetilde{C}^{1}(\bar{\Omega})\right)\right]^{3} .
\end{gathered}
$$

Clearly, the resulting vector function $U$ is a weakened solution of problem (2.2)-(2.5).

We complete the proof of the theorem by noting that the function defined in (3.10) still belongs to the space $C^{1, \alpha}(\bar{\Omega})$ (see [27]) also in the case when $f(x, y) \in L_{\infty}(\Omega)$ but not only if $f \in C^{\alpha}(\Omega)$ and $\sup _{(x, y) \in \Omega}|f(x, y)| \leq M$ (as in Theorem [29]) or $f \in C(\bar{\Omega})$ (as was assumed up to this point). However, we have to replace inequality (3.11) by

$$
\|\varphi\|_{C^{1, \alpha}(\bar{\Omega})} \leq C\|f\|_{L_{\infty}(\Omega)} .
$$

4. The proof of Theorems 2 and 3 . In this section, using the local-in-time existence of a weakened solution of problem (2.2)-(2.5) shown in Sect. 3, we prove that this solution exists for all $t \geq 0$ if the problem's data satisfy the following assumptions: the doping density $\rho(x, y)$ is close to unity in the $L_{\infty}(\Omega)$ norm, the initial data $U(0)$ are small in the norm $\left[\widetilde{C}^{1}(\bar{\Omega})\right]^{3}$, the absolute values of the constants $\widetilde{G}, \widetilde{B}$ and $\ln \widetilde{N}_{g}$ are small enough, and $U(0) \in\left[H^{2}(\Omega)\right]^{3}$. Moreover, under these conditions we prove Theorem 3; i.e., we rigorously justify the stabilization method [35] for problem (2.2)-(2.5). 
As in Sect. 3, we first assume that the function $\rho(x, y)$ is not piecewise constant but belongs to $C^{1}(\bar{\Omega})$. Together with problem (2.2)-(2.5) (or $(2.6)-(2.9)$ ) we also consider the following problem obtained from $(1.14)-(1.17)$ by the change of variables $(2.1)$ :

$$
\begin{aligned}
& \frac{\partial}{\partial t}(\varphi-\Delta \varphi)=\Delta \varphi-\beta e^{M(x)}\left(e^{\chi}-1\right)+\beta\left(\rho-e^{M(x)}\right)+Z^{\prime \prime}(x), \\
& \frac{\partial}{\partial t}(\sigma-\Delta \sigma)=\Delta \sigma-a_{1}|\nabla \sigma|^{2}-a_{2}(\nabla \sigma, \nabla \chi)-a_{2} \sigma_{x} M^{\prime}-a_{3}(\nabla \sigma, \nabla \varphi) \\
& \text { - } a_{3} \sigma_{x} Z^{\prime}-a_{4}(\nabla \chi, \nabla \varphi)-a_{4} \chi_{x} Z^{\prime}-a_{4} \varphi_{x} M^{\prime} \\
& -a_{4} Z^{\prime} M^{\prime}-a_{5}|\nabla \varphi|^{2}-2 a_{5} \varphi_{x} Z^{\prime}-a_{5}\left(Z^{\prime}\right)^{2}-b c \sigma \\
& \frac{\partial}{\partial t}(\chi-\Delta \chi)=\Delta \chi+|\nabla \chi|^{2}+2 \chi_{x} M^{\prime}+\left(M^{\prime}\right)^{2}-b_{1}|\nabla \sigma|^{2}-b_{2}(\nabla \sigma, \nabla \chi) \\
& -b_{2} \sigma_{x} M^{\prime}-b_{3}(\nabla \sigma, \nabla \varphi)-b_{3} \sigma_{x} Z^{\prime}-b_{4}(\nabla \chi, \nabla \varphi) \\
& -b_{4} \chi_{x} Z^{\prime}-b_{4} \varphi_{x} M^{\prime}-b_{4} M^{\prime} Z^{\prime}-b_{5}|\nabla \varphi|^{2} \\
& -2 b_{5} \varphi_{x} Z^{\prime}-b_{5}\left(Z^{\prime}\right)^{2}-\frac{\beta}{1+\sigma} e^{M(x)}\left(e^{\chi}-1\right) \\
& -\frac{\beta}{1+\sigma}\left(e^{M(x)}-\rho\right)-n c \sigma+M^{\prime \prime} \\
& \varphi=\sigma=\chi=0 \text { on } \Gamma_{0} \\
& (\mathbf{l}, \nabla \varphi)=(\mathbf{l}, \nabla \sigma)=(\mathbf{l}, \nabla \chi)=0 \text { on } \Gamma_{l} \text {; } \\
& \left\{\begin{array}{l}
\left.\varphi\right|_{t=0}=\varphi_{0}(x, y), \\
\left.\sigma\right|_{t=0}=\sigma_{0}(x, y), \\
\left.\chi\right|_{t=0}=\chi_{0}(x, y) .
\end{array}\right.
\end{aligned}
$$

The main idea of the proof of Theorem 2 is the derivation of a special differential inequality for the energy integral for system (2.2) [36].

We multiply the first equation of system (4.1) by $2 \varphi$, the second one by $2 \sigma$, and the third one by $2 \chi$. Likewise, we multiply the first equation of $(2.6)$ by $2 \theta_{\varphi}$, the second one by $2 \theta_{\sigma}$, and the third one by $2 \theta_{\chi}$ respectively. After simple algebra we obtain

$$
\begin{gathered}
\frac{\partial}{\partial t}\left(\varphi^{2}+|\nabla \varphi|^{2}\right)-2 \operatorname{div}\left(\varphi \nabla \varphi_{t}\right)-2 \operatorname{div}(\varphi \nabla \varphi)+2|\nabla \varphi|^{2} \\
=2 \varphi \beta e^{M(x)}\left(e^{\chi}-1\right)+2 \varphi \beta\left(\rho-e^{M(x)}\right)+2 \varphi Z^{\prime \prime} ; \\
\frac{\partial}{\partial t}\left(\sigma^{2}+|\nabla \sigma|^{2}\right)-2 \operatorname{div}\left(\sigma \nabla \sigma_{t}\right)-2 \operatorname{div}(\sigma \nabla \sigma)+2|\nabla \sigma|^{2}+2 b c \sigma^{2} \\
=2 \sigma\left\{-a_{1}|\nabla \sigma|^{2}-a_{2}(\nabla \sigma, \nabla \chi)-a_{2} \sigma_{x} Z^{\prime}-a_{3}(\nabla \sigma, \nabla \varphi)-a_{3} \sigma_{x} M^{\prime}\right. \\
-a_{4}(\nabla \chi, \nabla \varphi)-a_{4} \chi_{x} Z^{\prime}-a_{4} \varphi_{x} M^{\prime}-a_{4} M^{\prime} Z^{\prime}-a_{5}|\nabla \varphi|^{2}-2 a_{5} \varphi_{x} Z^{\prime} \\
\left.-a_{5}\left(Z^{\prime}\right)^{2}\right\} ; \\
\frac{\partial}{\partial t}\left(\chi^{2}+|\nabla \chi|^{2}\right)-2 \operatorname{div}\left(\chi \nabla \chi_{t}\right)-2 \operatorname{div}(\chi \nabla \chi)+2|\nabla \chi|^{2}+2 n c \chi \sigma \\
+\frac{2 \beta}{1+\sigma} e^{M(x)}\left(e^{\chi}-1\right) \chi=2 \chi\left\{|\nabla \chi|^{2}+2 \chi_{x} M^{\prime}+\left(M^{\prime}\right)^{2}-b_{1}|\nabla \sigma|^{2}\right. \\
-b_{2}(\nabla \sigma, \nabla \chi)-b_{2} \sigma_{x} M^{\prime}-b_{3}(\nabla \sigma, \nabla \varphi)-b_{3} \sigma_{x} Z^{\prime}-b_{4}(\nabla \chi, \nabla \varphi)
\end{gathered}
$$




$$
\begin{gathered}
-b_{4} \chi_{x} Z^{\prime}-b_{4} \varphi_{x} M^{\prime}-b_{4} M^{\prime} Z^{\prime}-b_{5}|\nabla \varphi|^{2}-2 b_{5} \varphi_{x} Z^{\prime}-b_{5}\left(Z^{\prime}\right)^{2} \\
\left.-\frac{\beta}{1+\sigma}\left(e^{M(x)}-\rho\right)+M^{\prime \prime}\right\} ; \\
\frac{\partial}{\partial t}\left(\theta_{\varphi}\right)^{2}+2\left(\theta_{\varphi}\right)^{2}-2 \theta_{\varphi} \varphi=2 \theta_{\varphi} \beta e^{M(x)}\left(1-e^{\chi}\right)+2 \theta_{\varphi} \beta\left(\rho-e^{M(x)}\right) \\
+2 \theta_{\varphi} Z^{\prime \prime} ; \\
\frac{\partial}{\partial t}\left(\theta_{\sigma}\right)^{2}+2\left(\theta_{\sigma}\right)^{2}-2 \theta_{\sigma} \sigma+2 b c \theta_{\sigma} \sigma=-2 \theta_{\sigma}\left(a_{1}|\nabla \sigma|^{2}\right. \\
+a_{2}(\nabla \sigma, \nabla \chi)+a_{2} \sigma_{x} Z^{\prime}+a_{3}(\nabla \sigma, \nabla \varphi)+a_{3} \sigma_{x} M^{\prime}+a_{4}(\nabla \chi, \nabla \varphi) \\
\left.+a_{4} \chi_{x} Z^{\prime}+a_{4} \varphi_{x} M^{\prime}+a_{4} Z^{\prime} M^{\prime}-a_{5}|\nabla \varphi|^{2}+2 a_{5} \varphi_{x} Z^{\prime}+a_{5}\left(Z^{\prime}\right)^{2}\right) ; \\
\frac{\partial}{\partial t}\left(\theta_{\chi}\right)^{2}+2\left(\theta_{\chi}\right)^{2}-2 \theta_{\chi} \chi+2 n c \theta_{\chi} \sigma+\frac{2 \beta \theta_{\chi}}{1+\sigma} e^{M(x)}\left(e^{\chi}-1\right) \\
=-2 \theta_{\chi}\left\{-|\nabla \chi|^{2}-2 \chi_{x} M^{\prime}-\left(M^{\prime}\right)^{2}+b_{1}|\nabla \sigma|^{2}+b_{2}(\nabla \sigma, \nabla \chi)+b_{2} \sigma_{x} M^{\prime}\right. \\
+b_{3}(\nabla \sigma, \nabla \varphi)+b_{3} \sigma_{x} Z^{\prime}+b_{4}(\nabla \chi, \nabla \varphi)+b_{4} \chi_{x} Z^{\prime}+b_{4} \varphi_{x} M^{\prime} \\
+b_{4} M^{\prime} Z^{\prime}+b_{5}|\nabla \varphi|^{2}+2 b_{5} \varphi_{x} Z^{\prime}+b_{5}\left(Z^{\prime}\right)^{2} \\
\left.+\frac{\beta}{1+\sigma}\left(e^{M(x)}-\rho\right)+M^{\prime \prime}\right\} .
\end{gathered}
$$

Taking into account the boundary conditions (4.2), (4.3), we integrate (4.5)-(4.10) over the rectangle $\Omega$. We multiply the obtained equalities by the constants $\alpha_{1}, \alpha_{2}, \ldots, \alpha_{6}$ respectively and sum the results. Then we get the following differential identity:

$$
\frac{d}{d t} \Phi(t)+\Phi_{1}(t)=\Phi_{2}(t)+\Phi_{3}(t),
$$

where

$$
\begin{gathered}
\Phi(t)=\int_{\Omega}\left\{\alpha_{1}\left(\varphi^{2}+|\nabla \varphi|^{2}\right)+\alpha_{2}\left(\sigma^{2}+|\nabla \sigma|^{2}\right)+\alpha_{3}\left(\chi^{2}+|\nabla \chi|^{2}\right)\right. \\
\left.+\alpha_{4}\left(\theta_{\varphi}\right)^{2}+\alpha_{5}\left(\theta_{\sigma}\right)^{2}+\alpha_{6}\left(\theta_{\chi}\right)^{2}\right\} d x d y \\
\Phi_{1}(t)=2 \int_{\Omega}\left\{\alpha_{1}|\nabla \varphi|^{2}+\alpha_{2}|\nabla \sigma|^{2}+\alpha_{2} b c \sigma^{2}+\alpha_{3}|\nabla \chi|^{2}+\alpha_{3} n c \chi \sigma\right. \\
+\alpha_{3} \frac{\beta \chi}{1+\sigma} e^{M(x)}\left(e^{\chi}-1\right)+\alpha_{4}\left(\theta_{\varphi}\right)^{2}-\alpha_{4} \theta_{\varphi} \varphi+\alpha_{5}\left(\theta_{\sigma}\right)^{2}-\alpha_{5} \theta_{\sigma} \sigma+\alpha_{5} b c \theta_{\sigma} \sigma \\
\left.+\alpha_{6}\left(\theta_{\chi}\right)^{2}-\alpha_{6} \theta_{\chi} \chi+\alpha_{6} n c \theta_{\chi} \sigma\right\} d x d y \\
\Phi_{2}(t)=2 \int_{\Omega}\left\{\alpha_{1} \beta \varphi e^{M(x)}\left(1-e^{\chi}\right)+\alpha_{1} \varphi\left(\beta\left(\rho-e^{M(x)}\right)+Z^{\prime \prime}\right)\right. \\
+K^{(1)}(\sigma, x) \sigma_{x} \sigma-\alpha_{2} a_{4} \chi_{x} \sigma Z^{\prime}+P^{(1)}(\sigma, x) \varphi_{x} \sigma+L^{(1)}(\sigma, x) \chi_{x} \chi \\
+G^{(1)}(\sigma, x) \chi \sigma_{x}+F^{(1)}(\sigma, x) \varphi_{x} \chi+E^{(1)}(\sigma, x) \sigma+H^{(1)}(\sigma, x) \chi \\
+K^{(2)}(\sigma, x) \sigma \theta_{\sigma}+P^{(2)}(\sigma, x) \varphi_{x} \theta_{\sigma}-\alpha_{5} a_{4} \chi_{x} \theta_{\sigma} Z^{\prime} \\
+L^{(2)}(\sigma, x) \chi_{x} \theta_{\chi}+G^{(2)}(\sigma, x) \sigma_{x} \theta_{\chi}+F^{(2)}(\sigma, x) \varphi_{x} \theta_{\chi} \\
+H^{(2)}(\sigma, x) \theta_{\varphi}+H^{(3)}(\sigma, x) \theta_{\sigma}-\alpha_{6} \frac{\beta}{1+\sigma} e^{M(x)}\left(e^{\chi}-1\right) \theta_{\chi}
\end{gathered}
$$




$$
\left.+H^{(4)}(\sigma, x) \theta_{\chi}\right\} d x d y
$$

and in (4.14) we use the following notation:

$$
\begin{aligned}
K^{(1)}(\sigma, x)= & -\alpha_{2}\left(a_{2} M^{\prime}+a_{3} Z^{\prime}\right), \\
P^{(1)}(\sigma, x)= & -\alpha_{2}\left(a_{4} M^{\prime}+2 a_{5} Z^{\prime}\right), \\
L^{(1)}(\sigma, x)= & \alpha_{3}\left(2 M^{\prime}-b_{4} Z^{\prime}\right), \\
G^{(1)}(\sigma, x)= & -\alpha_{3}\left(b_{2} M^{\prime}+2 b_{3} Z^{\prime}\right), \\
F^{(1)}(\sigma, x)= & -\alpha_{3}\left(b_{4} M^{\prime}+2 b_{5} Z^{\prime}\right), \\
E^{(1)}(\sigma, x)= & -\alpha_{2}\left(a_{4} M^{\prime} Z^{\prime}+a_{5}\left(Z^{\prime}\right)^{2}\right), \\
H^{(1)}(\sigma, x)= & -\alpha_{3}\left(b_{4} M^{\prime} Z^{\prime}+b_{5}\left(Z^{\prime}\right)^{2}+\frac{\beta}{1+\sigma}\left(e^{M(x)}-\rho\right)-M^{\prime \prime}-\left(M^{\prime}\right)^{2}\right), \\
K^{(2)}(\sigma, x)= & -\alpha_{5} M^{\prime}\left(a_{2}+a_{3}\right), \\
P^{(2)}(\sigma, x)= & -\alpha_{5}\left(a_{4} M^{\prime}+2 a_{5} Z^{\prime}\right), \\
L^{(2)}(\sigma, x)= & \alpha_{6}\left(2 M^{\prime}-b_{4} Z^{\prime}\right), \\
G^{(2)}(\sigma, x)= & -\alpha_{6}\left(b_{2} M^{\prime}+b_{3} Z^{\prime}\right), \\
F^{(2)}(\sigma, x)= & -\alpha_{6}\left(b_{4} M^{\prime}+2 b_{5} Z^{\prime}\right), \\
H^{(2)}(\sigma, x)= & \alpha_{4}\left(\beta e^{M(x)}\left(1-e^{\chi}\right)+\beta\left(\rho-e^{M(x)}\right)+Z^{\prime \prime}\right), \\
H^{(3)}(\sigma, x)= & -\alpha_{5}\left(a_{4} M^{\prime} Z^{\prime}+a_{5}\left(Z^{\prime}\right)^{2}\right), \\
H^{(4)}(\sigma, x)= & -\alpha_{6}\left(-\left(M^{\prime}\right)^{2}+b_{4} M^{\prime} Z^{\prime}+b_{5}\left(Z^{\prime}\right)^{2}+\frac{\beta}{1+\sigma}\left(e^{M(x)}-\rho\right)+M^{\prime \prime}\right), \\
& +\Phi_{3}(t)=-2 \int\left\{( \alpha _ { 2 } \sigma + \alpha _ { 5 } \theta _ { \sigma } ) \left(a_{1}|\nabla \sigma|^{2}+a_{2}(\nabla \sigma, \nabla \chi)\right.\right. \\
\left.+a_{3}(\nabla \sigma, \nabla \varphi)+a_{4}(\nabla \chi, \nabla \varphi)+a_{5}|\nabla \varphi|^{2}\right)+\left(\alpha_{3} \chi+\alpha_{6} \theta_{\chi}\right)\left(-|\nabla \chi|^{2}\right. & b_{2}(\nabla \sigma, \nabla \chi)+b_{3}(\nabla \sigma, \nabla \varphi)+b_{4}(\nabla \sigma, \nabla \varphi) \\
&
\end{aligned}
$$

It follows from Theorem 1 that if the initial data $U(0)$ belong to $\left[\widetilde{C}^{1}(\bar{\Omega})\right]^{3}$, then we can choose such a $T_{*}>0$ that for $0 \leq t \leq T_{*}$ there exists a weakened solution of problem (2.2)-(2.5) and, therefore, we can define the constant

$$
M_{*}=\max \left\{\max _{t \in\left[0, T_{*}\right]}\|\sigma(t)\|_{C(\bar{\Omega})}, \max _{t \in\left[0, T_{*}\right]}\|\chi(t)\|_{C(\bar{\Omega})}\right\}
$$

We first consider the limit case of inequality (4.11) when $\rho(x, y) \equiv 1, M(x)=Z(x) \equiv$ 0 . Then the integrals $\Phi(t)$ and $\Phi_{3}(t)$ stay the same, but the integrals $\Phi_{1}(t)$ and $\Phi_{2}(t)$ 
become simpler:

$$
\begin{gathered}
\Phi_{1}(t)=2 \int_{\Omega}\left\{\alpha_{1}|\nabla \varphi|^{2}+\alpha_{2}|\nabla \sigma|^{2}+\alpha_{2} b c \sigma^{2}+\alpha_{3}|\nabla \chi|^{2}\right. \\
+\alpha_{3} n c \chi \sigma+\alpha_{3} \frac{\beta \chi}{1+\sigma}\left(e^{\chi}-1\right)+\alpha_{4}\left(\theta_{\varphi}\right)^{2}-\alpha_{4} \theta_{\varphi} \varphi+\alpha_{5}\left(\theta_{\sigma}\right)^{2}-\alpha_{5} \theta_{\sigma} \sigma \\
\left.+\alpha_{5} b c \theta_{\sigma} \sigma+\alpha_{6}\left(\theta_{\chi}\right)^{2}-\alpha_{6} \theta_{\chi} \chi+\alpha_{6} n c \theta_{\chi} \sigma\right\} d x d y \\
\Phi_{2}(t)=2 \int_{\Omega}\left\{\alpha_{1} \beta \varphi\left(1-e^{\chi}\right)+\alpha_{4} \theta_{\varphi} \beta\left(1-e^{\chi}\right)\right. \\
\left.+\alpha_{6} \frac{\beta \theta_{\chi}}{1+\sigma}\left(1-e^{\chi}\right)\right\} d x d y
\end{gathered}
$$

Using the Friedrichs inequality (3.19), the Young inequality

$$
a b \leq \frac{1}{4 \varepsilon} a^{2}+\varepsilon b^{2}, a, b \in R, \varepsilon>0,
$$

and the evident inequality

$$
\left|e^{\chi}-1\right| \leq|\chi| e^{|\chi|}
$$

as in 36, we obtain the estimate

$$
\begin{gathered}
\Phi_{1}(t)-\Phi_{2}(t) \geq \int_{\Omega}\left\{\alpha_{1}|\nabla \varphi|^{2}+\alpha_{2}|\nabla \sigma|^{2}+\alpha_{3}|\nabla \chi|^{2}\right. \\
+2(Y, A Y)\} d x d y \geq M_{1} \Phi(t),
\end{gathered}
$$

where the vector $Y$ and the matrix $A$ are the following:

$$
\left(\begin{array}{cccccc}
\alpha_{1}(1-\beta \varepsilon) & 0 & 0 & -\frac{1}{2} \alpha_{4} & 0 & 0 \\
0 & \alpha_{2}(1+b c) & \frac{1}{2} \alpha_{3} n c & 0 & -\frac{1}{2} \alpha_{5}(1-b c) & \frac{1}{2} \alpha_{6} n c \\
0 & \frac{1}{2} \alpha_{3} n c & \mathcal{A} & 0 & 0 & -\frac{1}{2} \alpha_{6} \\
-\frac{1}{2} \alpha_{4} & 0 & 0 & \alpha_{4}(1-\beta \varepsilon) & 0 & 0 \\
0 & -\frac{1}{2} \alpha_{5}(1-b c) & 0 & 0 & \alpha_{5} & 0 \\
0 & \frac{1}{2} \alpha_{6} n c & -\frac{1}{2} \alpha_{6} & 0 & 0 & \alpha_{6}\left(1-\frac{\beta \varepsilon}{1-M_{*}}\right)
\end{array}\right)
$$

$\mathcal{A}=\alpha_{3}-\left(\alpha_{1}+\alpha_{4}+\frac{\alpha_{6}}{1-M_{*}}\right) \frac{e^{2 M_{*}}}{4 \varepsilon} \beta$, and $M_{1}$ and $\varepsilon$ are positive constants.

Choosing the constants $\alpha_{1}, \ldots, \alpha_{6}$ as $\alpha_{1}=\alpha_{4}=\alpha_{6}=\varepsilon^{2}, \alpha_{3}=1, \alpha_{5}=\varepsilon, \alpha_{2}=\frac{1}{\varepsilon}(\varepsilon$ is small enough), we can guarantee the fulfillment of inequality (4.20) (in this case the matrix $A>0$ because for small $\sigma$ we have $b(\sigma) c(\sigma)>0[20]$ ). The constant $M_{1}$ actually depends on $M_{*}$ and $\varepsilon$.

For estimating the integral $\Phi_{3}(t)$ we use the Gagliardo-Nirenberg inequality [37]

$$
\|\nabla u\|_{L_{4}(\Omega)}^{2} \leq C(\Omega)\|u\|_{L_{\infty}(\Omega)}\|u\|_{H^{2}(\Omega)}
$$

the Sobolev inequality 38

$$
\|u\|_{C(\bar{\Omega})} \leq M_{b}\|u\|_{H^{2}(\Omega)}
$$

where $M_{b}$ is the best embedding constant, and the Young inequality (4.19) mentioned above. 
Then, for example, the following inequalities hold:

$$
\begin{aligned}
\left.\left|\int_{\Omega} a_{1} \sigma\right| \nabla \sigma\right|^{2} d x d y \mid & \leq M_{a_{1}} \max _{(x, y) \in \bar{\Omega}}|\sigma(t, x, y)| \int_{\Omega}|\nabla \sigma|^{2} d x d y \\
& \leq M_{a_{1}} \widetilde{M}_{b}[\Phi(t)]^{\frac{3}{2}}
\end{aligned}
$$

where the constant $M_{a_{1}}$ estimates the function $a_{1}(\sigma)$ for $0 \leq|\sigma| \leq M_{*}$, the constant $\widetilde{M}_{b}$ is connected with the embedding constant $M_{b}$ and the integral $\Phi(t)$, i.e., it depends on $\varepsilon$

$$
\begin{gathered}
\left|\int_{\Omega} a_{2} \sigma(\nabla \sigma, \nabla \chi)^{2} d x d y\right| \leq M_{a_{2}} \max _{(x, y) \in \bar{\Omega}}|\sigma(t, x, y)| \\
\cdot\left(\int_{\Omega}|\nabla \sigma|^{2} d x d y+\int_{\Omega}|\nabla \chi|^{2} d x d y\right) \leq M_{a_{2}} \widetilde{M}_{c}[\Phi(t)]^{\frac{3}{2}} .
\end{gathered}
$$

At last,

$$
\begin{aligned}
\left.\left|\int_{\Omega} a_{1} \theta_{\sigma}\right| \nabla \sigma\right|^{2} d x d y \mid & \leq M_{a_{1}}\left(\int_{\Omega}\left|\theta_{\sigma}\right|^{2} d x d y\right)^{\frac{1}{2}}\left(\int_{\Omega}|\nabla \sigma|^{4} d x d y\right)^{\frac{1}{2}} \\
& \leq M_{a_{1}} \widetilde{M}_{d}[\Phi(t)]^{\frac{3}{2}} .
\end{aligned}
$$

Taking into account inequalities (4.21)-(4.23), we derive the desired estimate

$$
\left|\Phi_{3}(t)\right| \leq M_{2}[\Phi(t)]^{\frac{3}{2}}
$$

where the constant $M_{2}$ depends on $M_{*}, \varepsilon, C(\Omega)$ and the embedding constant $M_{b}$ (we recall that the constants $C(\Omega)$ and $M_{b}$ appear in the Gagliardo-Nirenberg and Sobolev inequalities).

With the reference to (4.20) and (4.24), from inequality (4.11) we obtain the differential inequality

$$
\frac{d}{d t} \Phi(t)+M_{1} \Phi(t) \leq M_{2} \Phi(t)^{\frac{3}{2}}
$$

REMARK 4.1. In the present work we do not want to find an optimal value of $\varepsilon$ (for a fixed $M_{*}$ ), i.e., such a number that $M_{1}$ is maximal and vice versa the constant $M_{2}$ is minimal. This is a separate problem.

It is clear that if the function $\rho(x, y)$ is close to unity and the constants $\widetilde{G}, \widetilde{B}$ and $\ln \widetilde{N}_{g}$ are small in the sense described in the beginning of this section, then, in view of (4.15), instead of inequality (4.25) we can get the relation

$$
\frac{d}{d t} \Phi(t)+\widetilde{M}_{1} \Phi(t) \leq M_{2} \Phi(t)^{\frac{3}{2}}+K \Phi(t)^{\frac{1}{2}},
$$

where the positive constants $\widetilde{M}_{1}$ and $K$ depend on the constants $\widetilde{G}, \widetilde{B}, \ln \widetilde{N}_{g}, \varepsilon, M_{*}$ and the function $\rho(x, y)$.

REMARK 4.2. While deriving estimate (4.26) we assumed that each component of the unknown function $U(t, x, y)(\varphi(t, x, y), \sigma(t, x, y)$ and $\chi(t, x, y))$ belongs to the space $C^{1}\left([0, T], H^{2}(\Omega)\right)$. This can be guaranteed by a suitable choice of the initial data. Indeed, let $U(0) \in\left[C^{1, \alpha}(\bar{\Omega})\right]^{3}$ (relations (2.3), (2.4) are satisfied as well). Then, in view of (3.23) and Theorem [29] formulated in Sect. 3, $U(0) \in\left[C^{1, \alpha}(\bar{\Omega})\right]^{3}$ (as is noted in [29, this result is true under the more general assumption that $f \in L_{\infty}(\Omega)$ ). Moreover, $\alpha$ should satisfy 


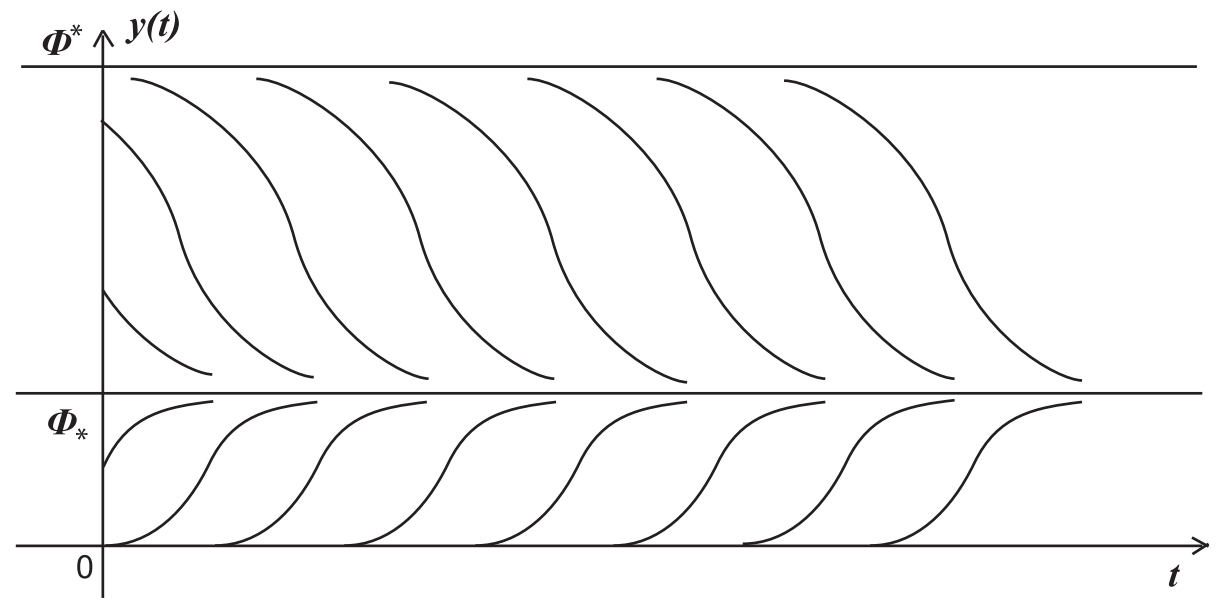

FIG. 3. Qualitative behavior of solutions of equation (4.27).

only the requirement $0<\alpha<1$. Assume that $U(0) \in\left[H^{2}(\Omega)\right]^{3}$. In this case, using (3.23), (3.10) and the well-known Calderon-Zygmund inequality [27] (here we use it for estimating in $H^{2}(\Omega)$ the potential of area, i.e., the first term in (3.10)), we obtain that $U(t, x, y) \in\left[C^{1}\left([0, T], H^{2}(\Omega)\right)\right]^{3}$.

Let us analyze the behavior of the solutions of the equation

$$
\frac{d y}{d t}=M_{2} y^{\frac{3}{2}}-\widetilde{M}_{1} y+K y^{\frac{1}{2}}
$$

in the neighborhood of the constant solutions

$$
\begin{gathered}
y \equiv 0, \\
y(t) \equiv \Phi_{*}=\frac{1}{2 M_{2}}\left(\widetilde{M}_{1}-\sqrt{\widetilde{M}_{1}^{2}-4 M_{2} K}\right), \\
y(t) \equiv \Phi^{*}=\frac{1}{2 M_{2}}\left(\widetilde{M}_{1}+\sqrt{\widetilde{M}_{1}^{2}-4 M_{2} K}\right) .
\end{gathered}
$$

It is clear that for $0 \leq y(t)<\Phi^{*}$ the remaining solutions of equation (4.27) (which can be found from the initial data in an explicit form) behave qualitatively as the time $t$ increases as is shown in Figure 3 .

We note that if the initial data $y(0)$ lies between $\Phi_{*}$ and $\Phi^{*}\left(\Phi_{*}<y(0)<\Phi^{*}\right)$, then the corresponding solution $y(t)$ asymptotically decays to $\Phi_{*}$ as $t \rightarrow+\infty$, but if $0 \leq y(0)<\Phi_{*}$, then $y(t)$ asymptotically increases to $\Phi_{*}$ as $t \rightarrow+\infty$.

Then, in view of the Sobolev inequality, inequality (4.26) and the behavior of the solutions of equation (4.27) as $t \rightarrow+\infty$, we have the following relations:

$$
\begin{gathered}
\left\|\sigma\left(T_{*}\right)\right\|_{C(\bar{\Omega})} \leq M_{b}\left\|\sigma\left(T_{*}\right)\right\|_{H^{2}(\Omega)} \leq M_{b} M_{3}\left(\Phi\left(T_{*}\right)\right)^{\frac{1}{2}} \\
\leq M_{b} M_{3}\left(\Phi^{*}\right)^{\frac{1}{2}}
\end{gathered}
$$

where $M_{3}>0$ is a constant dependent on $M_{*}$ and $\varepsilon$. 
It is clear that if the inequality

$$
M_{b} M_{3}\left(\Phi_{*}\right)^{\frac{1}{2}} \leq M_{*}
$$

holds which, in view of (4.28), for a given $M_{*}$ is a restriction on the constant $K$, then we can always choose such initial data that

$$
\left\|\sigma\left(T_{*}\right)\right\|_{C(\bar{\Omega})} \leq M_{*}
$$

Hence, as new initial data we should take $\varphi\left(T_{*}, x, y\right), \sigma\left(T_{*}, x, y\right), \chi\left(T_{*}, x, y\right)$. Continuing this process, we find a solution on the segment $\left[T_{*}, 2 T_{*}\right]$.

That is, reasoning as above, we construct a solution for all $0 \leq t<+\infty$. Moreover, it obeys the estimate

$$
\Phi(t) \leq \Phi^{*}, 0 \leq t<+\infty
$$

Thus, under the restrictions described in the beginning of this section and the additional assumption that the doping density $\rho(x, y)$ belongs to $C^{1}(\bar{\Omega})$ and $U(0) \in\left[C^{1, \alpha}(\bar{\Omega})\right]^{3}$ (relations (2.3), (2.4) hold for the initial data) Theorem 2 is proved. In the end of this section we will get an analogous result but for the weakened solution of problem (2.2)-(2.5) and in the case when the function $\rho(x, y)$ is piecewise constant and $U(0) \in\left[\widetilde{C}^{1}(\bar{\Omega})\right]^{3} \cap\left[H^{2}(\Omega)\right]^{3}$. Now we pass to the proof of Theorem 3 .

In view of inequality (4.31), the differential inequality (4.26) and the Arzelà-Ascoli theorem [34, the family of functionals $\Phi(t)$ is compact in $C[0, T]$. This family is formed by the set of functions $\varphi(t, x, y), \sigma(t, x, y)$ and $\chi(t, x, y)$, and each triplet of functions uniquely defined by the initial data $\left.\varphi\right|_{t=0},\left.\sigma\right|_{t=0},\left.\chi\right|_{t=0}$.

Hence, any sequence of functionals $\left\{\Phi_{U_{n}}(t)\right\}_{n \in N}$ ( $N$ is the set of natural numbers) contains a subsequence $\left\{\Phi_{U_{n_{k}}}(t)\right\}_{k \in N}$ converging to a continuous on $[0, T]$ function $\widehat{\Phi}(t)$.

For a fixed $0 \leq t^{*} \leq T$ we consider a bounded in $\left[H^{2}(\Omega)\right]^{3}$ set of vector functions $\left\{U_{n_{k}}\left(t^{*}, x, y\right)\right\}_{k \in N}$ under the condition $\Phi_{U_{n_{k}}}\left(t^{*}\right) \leq \Phi^{*}, k \in N$. Thanks to the compactness of the embedding of $H^{2}(\Omega)$ to $H^{1}(\Omega)$ [32] there exists a subsequence $U_{\widetilde{n}_{k}}$ converging in $\left[H^{1}(\Omega)\right]^{3}$ to a certain vector function $U^{*}\left(t^{*}, x, y\right)$. In view of the reflexivity of the space $H^{2}(\Omega)$ and the boundedness of the set of functionals $\left\{\Phi_{U_{\widetilde{n}_{k}}}\left(t^{*}\right)\right\}_{k \in N}$ [39], we have that $U^{*}\left(t^{*}, x, y\right) \in\left[H^{2}(\Omega)\right]^{3}$ and $U_{\widetilde{n}_{k}}\left(t^{*}, x, y\right) \rightarrow U^{*}\left(t^{*}, x, y\right)$ weakly in $\left[H^{2}(\Omega)\right]^{3}$. Moreover, $\Phi_{U^{*}}(t) \leq \Phi^{*}$ if $0 \leq t<+\infty$.

The last result can be essentially improved. Namely, we can prove strong convergence, i.e., the convergence in $\left[H^{2}(\Omega)\right]^{3}$ of the subsequence $U_{\widetilde{n}_{k}}(t, x, y)$ to a limit function $U^{*}(t, x, y)$ for $0 \leq t \leq T$. However, probably it should be necessary to specify the choice of this subsequence.

Consider the new functional

$$
\begin{gathered}
\widetilde{\Phi}_{U_{1}, U_{2}}(t)=\int_{\Omega}\left\{\alpha_{1}\left(\left(\varphi_{1}-\varphi_{2}\right)^{2}+\left|\nabla \varphi_{1}-\nabla \varphi_{2}\right|^{2}\right)+\alpha_{2}\left(\left(\sigma_{1}-\sigma_{2}\right)^{2}\right.\right. \\
\left.+\left|\nabla \sigma_{1}-\nabla \sigma_{2}\right|^{2}\right)+\alpha_{3}\left(\left(\chi_{1}-\chi_{2}\right)^{2}+\left|\nabla \chi_{1}-\nabla \chi_{2}\right|^{2}\right)+\alpha_{4}\left(\theta_{\varphi_{1}}-\theta_{\varphi_{2}}\right)^{2} \\
\left.+\alpha_{5}\left(\theta_{\sigma_{1}}-\theta_{\sigma_{2}}\right)^{2}+\alpha_{6}\left(\theta_{\chi_{1}}-\theta_{\chi_{2}}\right)^{2}\right\} d x d y .
\end{gathered}
$$

Reasoning by analogy with the derivation of inequality (4.26) and taking into account that the initial data are actually defined by the assignment of gradients of the functions $\varphi_{0}(x, y), \sigma_{0}(x, y), \chi_{0}(x, y)$ (see the proof of Theorem 1 ) and, therefore, there is a 


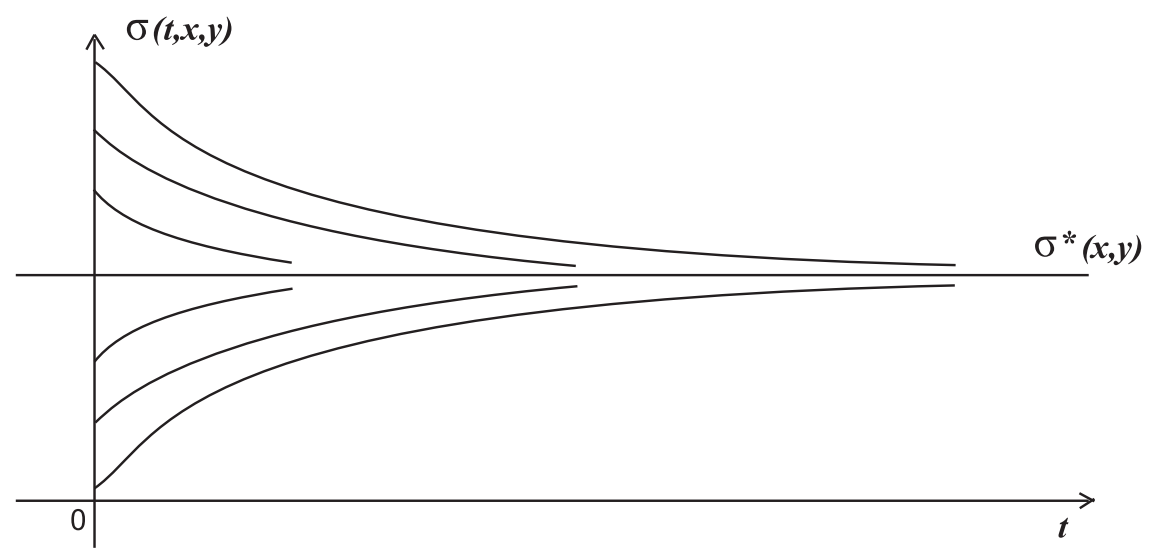

FIG. 4. Asymptotic behavior of the function $\sigma(t, x, y)$ as $t \rightarrow+\infty$.

possibility to diminish them, we get the differential inequality

$$
\frac{d}{d t} \widetilde{\Phi}_{U_{1}, U_{2}}+M_{3} \widetilde{\Phi}_{U_{1}, U_{2}} \leq 0,
$$

where $M_{3}>0$ and $M_{3}$ again depends on $\varepsilon$ and $M_{*}$. Integrating inequality (4.33), we obtain

$$
\widetilde{\Phi}_{U_{1}, U_{2}}(t) \leq e^{-M_{3} t} \widetilde{\Phi}_{U_{1}, U_{2}}(0) .
$$

Then, assuming additionally that $U^{*}(0, x, y) \in\left[H^{2}(\Omega)\right]^{3}$, we can choose a subsequence of initial data $U_{n}(0, x, y)$ converging in $\left[H^{2}(\Omega)\right]^{3}$ to $U^{*}(0, x, y)$. For this purpose it is enough to use, for example, a mollification process [38. For sufficiently smooth initial data $U_{n}(0, x, y)$ we can construct a unique solution from $\left.C^{1}\left([0, T], H^{2}(\Omega)\right)\right]^{3}$. In view of estimate (4.34), the sequence of solutions $U_{n}(t, x, y)$ converges to a certain vector function $U^{*}(t, x, y)$ in $\left.C\left([0, T], H^{2}(\Omega)\right)\right]^{3}$.

Clearly, if we consider the whole life span $0 \leq t<+\infty$ of solutions, then estimate (4.34) implies that all the solutions of problem (2.2)-(2.5) converge to the unique vector function $U^{*}(t, x, y)$.

If the initial data are chosen from $C^{1, \alpha}(\bar{\Omega})$ (see Remark 4.2), then the limit function is the solution of problem $(2.2)-(2.5)$ belonging to $\left[C^{1}\left([0, T], C^{1, \alpha}(\bar{\Omega})\right)\right]^{3}$. On the other hand, using the fact that the solution does not leave the closed ball of location of the initial data, the autonomy of system (2.2) and the Schauder fixed-point theorem [27], we can prove, as in the case of an autonomous ODE system, the existence of at least one stationary solution of problem (2.2)-(2.4) 33. In view of estimate (4.34), this solution is unique. Hence, if the initial data are located in a possibly small neighborhood of the origin in $C^{1, \alpha}(\bar{\Omega})$, then all the solutions of problem (2.2)-(2.5) for these initial data converge as $t \rightarrow \infty$ to the vector function $U^{*}(x, y)$ which is the stationary solution of problem (2.2)-(2.4).

Figure 4 illustrates the behavior of solutions as $t$ tends to $+\infty$. For definiteness only one of the components of the vector $U(t, x, y)$ is drawn for various initial data and a fixed 
point $(x, y)$. Namely, this is the component $\sigma(t, x, y)$ (for a fixed $(x, y)$ ) and $\sigma^{*}(x, y)$ is the corresponding component of the stationary solution $U^{*}(x, y)$.

The fact that, in view of representation (3.4), the components $\varphi, \sigma$ and $\chi$ of the weakened solution of problem $(2.2)-(2.5)$ belong to the space $C^{1}\left([0, T], H^{2}(\Omega)\right)$ also in the case when the doping density $\rho(x, y)$ is a piecewise constant function (see (1.4)) completes the proof of Theorems 2 and 3. Therefore, the functionals $\Phi(t), \Phi_{i}(t), i=$ $1,2,3$, already used in this section are defined on this solution. Hence, by analogy with the case $\rho(x, y) \in C^{1}(\bar{\Omega})$ studied above we get Theorems 2 and 3 .

REMARK 4.3. Theorems 2 and 3 for the same assumptions on the function $\rho(x, y)$ and the parameters $\widetilde{G}, \widetilde{B}$ and $\ln \widetilde{N}_{g}$ imply the solvability in $C^{1, \alpha}(\bar{\Omega})$ of the following elliptic problem:

$$
\left\{\begin{array}{l}
\varphi-\Delta \varphi=\mathcal{F}^{\varphi}(\chi, \rho), \\
\sigma-\Delta \sigma=\mathcal{F}^{\sigma}(\nabla \sigma, \nabla \chi, Q, \sigma), \\
\chi-\Delta \chi=\mathcal{F}^{\chi}(\nabla \sigma, \nabla \chi, Q, \sigma, \chi, \rho),
\end{array} \quad(x, y) \in \Omega,\right.
$$

where the functions $\chi, \sigma$ and $\varphi$ satisfy the boundary conditions (1.13).

\section{REFERENCES}

[1] S. Selberherr, Analysis and Simulation of Semiconductor Devices, Springer-Verlag, New York, Vienna, 1984.

[2] W. Hansch, The drift diffusion equations and its applications in MESFET modeling, SpringerVerlag, Vienna, 1991.

[3] P. Markowich, C.A. Ringhofer, and C. Schmeiser, Semiconductor equations, Springer-Verlag, Vienna, 1990. MR1063852 (91j:78011)

[4] D. Chen, E.C. Kan, U. Ravaioli, C-W. Shu, and R. Dutton, An improved energy-transport model including nonparabolicity and non-Maxwellian distribution effects, IEEE on Electron Device Letters, 13 (1992) pp.26-28.

[5] E. Lyumkis, B. Polsky, A. Shir, and P. Visocky, Transient semiconductor device simulation including energy balance equation, COMPEL, 11 (1992), pp. 311-325.

[6] N.B. Abdallah, P. Degond, On a hierarchy of macroscopic models for semiconductors, J. Math. Phys. 37 (1996), pp. 3308-3333. MR1401227(98b:82091)

[7] A.M. Anile, V. Romano, Hydrodynamical modeling of charge carrier transport in semiconductors, MECCANICA, 35 (2000), pp. 249-296.

[8] A.M. Anile, G. Mascali, and V. Romano, Recent developments in hydrodynamical modeling of semiconductors, Lecture Notes in Mathematics, no. 1823, Springer, 2003. MR2073497

[9] A.M. Anile, V. Romano, Non-parabolic band transport in semiconductors: closure of the moment equations, Cont. Mech. Thermodyn., 11 (1999), pp. 307-325. MR.1723705 (2000j:82044)

[10] V. Romano, Non-parabolic band transport in semiconductors: closure of the production terms in the moment equations, Cont. Mech. Thermodyn., 12 (2000), pp. 31-51. MR.1753005 (2001g:82117)

[11] I. Müller, T. Ruggeri, Rational Extended Thermodynamics, Springer-Verlag, Berlin, 1998. MR 1632151(99h:80001)

[12] D. Jon, J. Casas-Vazques, and G. Lebon, Extended irreversible thermodynamics, Springer-Verlag, Berlin, 1993. MR.1271780(95a:80004)

[13] C.D. Levermore, Moment Closure Hierarchies for Kinetic Theories, J. Stat. Phys., 83 (1996), pp. 331-407. MR 1392419 (97e:82041)

[14] A.M. Anile, O. Muscato, and V. Romano, Moment Equations with maximum entropy closure for carrier transport in semiconductor devices: validation in bulk silicon, VLSI Design 10 (2000), pp. 335-354.

[15] O. Muscato, V. Romano, Simulation of submicron silicon diode with a non-parabolic hydrodynamical model based on the maximum entropy principle, VLSI Design 13 (2001), pp. 273-279.

[16] V. Romano, Nonparabolic band hydrodynamical model of silicon semiconductors and simulation of electron devices, Math. Meth. Appl. Sci., 24 (2001), pp. 439-471. MR1829038 (2002c:82083) 
[17] V. Romano, 2D simulation of a silicon MESFET with a non-parabolic hydrodynamical model based on the maximum entropy principle, J. Comp. Phys., 176 (2002), pp. 70-92.

[18] V. Romano, 2D numerical simulation of the MEP energy-transport model with a finite difference scheme, J. Comp. Phys., 221 (2007), pp. 439-468. MR2293138(2008b:82083)

[19] A.M. Blokhin, R.S. Bushmanov, A.S. Rudometova, and V. Romano, Linear asymptotic stability of the equilibrium state for the 2D MEP hydrodynamical model of charge transport in semiconductors, Nonlinear Analysis, 65 (2006), pp. 1018-1038. MR.2232491 (2006m:76153)

[20] A.M. Blokhin, R.S. Bushmanov, and V. Romano, Nonlinear asymptotic stability of the equilibrium state for the MEP model of charge transport in semiconductors, Nonlinear Analysis, 65 (2006), pp. 2169-2191. MR2266431 (2007i:35225)

[21] A.M. Blokhin, A.S. Ibragimova, and B.V. Semisalov, Construction of a computational algorithm for a system of moment equations describing charge transfer in semiconductors, Mathematical Modelling, 21:4 (2009), pp. 15-34 (in Russian). MR.2547344 (2010e:82111)

[22] A.M. Blokhin, A.S. Ibragimova, Numerical method for 2D Simulation of a Silicon MESFET with a Hydrodynamical Model Based on the Maximum Entropy Principle, SIAM Journal on Scientific Computing, 31:3 (2009), pp. 2015-2046. MR.2516142 (2010i:82188)

[23] J. L. Lions, E. Magenes, Non-homogeneous boundary value problems and applications, Springer, New York, 1972.

[24] M.A. Shubin, Pseudodifferential operators and spectral theory, Nauka, Moscow, 1978 (in Russian). MR509034 (80h:47057)

[25] G.V. Demidenko, S.V. Uspenskij, Embedding theorems and their applications to differential equations, Nauka, Novosibirsk, 1984 (in Russian).

[26] O.A. Ladyzhenskaya, N.N. Ural'tseva, Linear and quasilinear elliptic equations, Academic Press, New York, 1968. MR0244627 (39:5941)

[27] D. Gilbarg, N.S. Trudinger, Elliptic partial differential equations of second order, Springer-Verlag, Berlin, 1977. MR0473443 (57:13109)

[28] A.G. Sveshnikov, A.B. Alshin, M.O. Korpusov, and Yu.D. Pletner, Linear and nonlinear Sobolevtype equations, Moscow, Fizmatlit, 2007 (in Russian).

[29] A.M. Blokhin, D.L. Tkachev, Representation of the solution to a model problem in semiconductor physics, J. Math. Anal. Appl. 341 (2008), 1468-1475. MR2398541 (2008m:35048)

[30] M.A. Lavrentjev, B.V. Shabat, Methods of Theory of Complex-Valued Functions, Gos. Izdatelstvo Tekhniko-Teoreticheskoj Literatury, Moscow-Leningrad, 1951 (in Russian).

[31] H. Ghaevsky, K. Grier, and K. Zakharias, Nonlinear Operator Equations and Operator Differential Equations, Mir, Moscow, 1978 (in Russian). MR0636413 (58:30524b)

[32] S. Mizohata, The theory of partial differential equations, Cambridge University Press, New York, 1973. MR0599580 (58:29033)

[33] I.G. Petrovsky, Lectures on Partial Differential Equations, Dover Publications, New York, 1991. MR 1160355 (93a:35001)

[34] L.V. Kantorovich, G.P. Akilov, Functional Analysis, Pergamon Press, Oxford, 1982. MR664597 (83h:46002)

[35] K.I. Babenko, Fundamentals of numerical analysis, Moscow-Izhevsk, Regular and chaotic dynamics, 2002 (in Russian).

[36] A.M. Blokhin, A.S. Ibragimova, 1D Numerical Simulation of the MEP Mathematical Model in ballistic diode problem, Journal of Kinetic and Related Models, 2:1 (2009), 81-107. MR2472150 (2010e:82110)

[37] O.V. Besov, V.P. Il'in, S.M. Nikol'skiǔ, Integral representations of functions and embedding theorems, Moscow, Fizmatlit, 1996 (in Russian). MR.1450401 (98b:46037)

[38] S.L. Sobolev, Applications of functional analysis in mathematical physics. Translations of Mathematical Monographs, vol. 7, American Mathematical Society, Providence, 1963. MR0165337 $(29: 2624)$

[39] K. Iosida, Functional Analysis, Springer-Verlag, Berlin-New York, 1980. MR617913 (82i:46002) 